FACULDADE DE MEDICINA DE RIBEIRÃO PRETO DA UNIVERSIDADE DE SÃO PAULO

PEDRO HENRIQUE MARTE DE ARRUDA SAMPAIO

Assimetrias no exame neurológico de crianças com síndrome de Guillain-Barré

Ribeirão Preto 
PEDRO HENRIQUE MARTE DE ARRUDA SAMPAIO

\section{Assimetrias no exame neurológico de crianças com síndrome de Guillain-Barré}

Dissertação apresentada ao Departamento de Neurociências e Ciências do Comportamento da Faculdade de Medicina de Ribeirão Preto da Universidade de São Paulo para obtenção de título de Mestre em Neurologia.

Área de Concentração: Neurologia

Orientador: Prof. Dr. Amilton Antunes Barreira

Versão corrigida. A versão original encontra-se disponível tanto na Biblioteca da Unidade que aloja o Programa, quanto na Biblioteca Digital de Teses e Dissertações da USP (BDTD).

Ribeirão Preto 
Autorizo a reprodução e divulgação total ou parcial deste trabalho, por qualquer meio convencional ou eletrônico, para fins de estudo e pesquisa, desde que citada a fonte.

\section{FICHA CATALOGRÁFICA}

Sampaio, Pedro Henrique Marte de Arruda

Assimetrias no exame neurológico de crianças com síndrome de GuillainBarré. / Pedro Henrique Marte de Arruda Sampaio. - Ribeirão Preto, 2017.

62f.: $30 \mathrm{~cm}$

Dissertação (Mestrado Profissional) - Faculdade de Medicina de Ribeirão Preto da Universidade de São Paulo. Programa de Neurologia.

Orientador: Amilton Antunes Barreira.

1. Síndrome de Guillain-Barré 2. Síndrome de Miller Fisher 3. Assimetria 4. Epidemiologia 


\section{FOLHA DE APROVAÇÃO}

Nome: SAMPAIO, Pedro Henrique Marte de Arruda

Título: Assimetrias no exame neurológico de crianças com síndrome de Guillain-Barré

Dissertação apresentada à Faculdade de Medicina de Ribeirão Preto da Universidade de São Paulo para obtenção do título de Mestre em Neurologia.

Área de concentração: Neurologia / Neurociências

Aprovado em:

Banca Examinadora

Prof. Dr.: Instituição:

Julgamento: Assinatura:

Prof. Dr.: Instituição:

Julgamento: Assinatura:

Prof. Dr.: Instituição:

Julgamento: Assinatura: 


\section{AGRADECIMENTOS}

Aos funcionários do Serviço de Arquivo Médico que sempre foram muito compreensivos e solícitos durante a revisão dos prontuários.

À Faculdade de Medicina de Ribeirão Preto e ao Hospital das Clínicas da Faculdade de Medicina de Ribeirão Preto da Universidade de São Paulo pela oportunidade de realização do Mestrado Profissional.

Aos meus amigos e colegas de residência Ana Luiza, Breno, Camila, Nathalia e Rodrigo, pelo companheirismo e paciência no dia-a-dia.

Aos meus preceptores Carolina, André, Patrícia, Pedro e Vanessa por todo o apoio e auxílio que me deram.

Aos meus professores Claudia Ferreira da Rosa Sobreira e Wilson Marques Junior, por todo o aprendizado ao longo desses anos.

Ao meu mestre e mentor Amilton Antunes Barreira por todos os ensinamentos nesses anos todos e por ter confiado nas minhas habilidades.

Aos meus pais, que desde pequeno me inspiraram tanto a seguir o caminho da neurologia e da ciência; obrigado por tudo o que sempre fizeram por mim.

Aos meus irmãos Cadu e Guto, meus avós e a todos os meus familiares, que sempre me ajudaram tanto nas minhas escolhas da vida.

À minha amada e companheira Cris, esposa maravilhosa e que sempre foi a minha fonte de razão e força de vontade para percorrer essa longa jornada. 
"O mundo é um contínuo marulhar

Pequenos peixes cantam e dançam, nadam espertamente ao sabor das ondas que vêm e vão. Quem no entanto é capaz de saber o que se passa nas recônditas profundezas desse mar sem fim?

Quem algum dia já mediu sua exata profundidade?”

Eiji Yoshikawa, Musashi 


\section{RESUMO}

SAMPAIO, PHMA. Assimetrias no exame neurológico de crianças com síndrome de Guillain-Barré. 2017. 62f. Dissertação de mestrado. Faculdade de Medicina de Ribeirão Preto, Universidade de São Paulo, São Paulo, 2017.

A Síndrome de Guillain-Barré (SGB) é uma neuropatia periférica inflamatória aguda que tem sido definida pelo achado ou história de tetraparesia flácida arreflexa ascendente. Apresentações atípicas podem ser mais frequentes do que tem sido referido na literatura, particularmente na faixa etária infantil. Objetivo: Avaliar dados epidemiológicos e a prevalência de assimetria no exame neurológico em crianças com SGB. Métodos: Foram revisados 40 prontuários de crianças de 0 a 15 anos de idade com o diagnóstico de SGB, atendidas entre janeiro de 2000 e agosto de 2016. Avaliouse a presença de assimetrias no exame neurológico na admissão hospitalar, os desfechos clínicos e as características demográficas e clinico-laboratoriais. Resultados: Dois pacientes apresentaram assimetria no exame neurológico na admissão hospitalar e três pacientes admitidos com tetraparesia simétrica apresentaram um quadro motor assimétrico antes da internação. Uma criança evoluiu para assimetria após ter sido admitida com quadro simétrico. Outros oito casos tinham fraqueza segmentar. A presença de assimetria motora ou fraqueza segmentar se correlacionou com a progressão estática dos sintomas $(\mathrm{p}=0,004)$ e observou-se uma tendência desses pacientes serem mais jovens, mas essa diferença não foi significativa $(p=0,08)$. Onze pacientes apresentavam reflexos miotáticos preservados e um paciente exibia hiperreflexia na admissão hospitalar. A maioria dos pacientes foi admitida sem conseguir deambular e, na alta, a maioria deambulava com ou sem apoio. Cinco crianças necessitaram de suporte ventilatório e nenhuma foi a óbito. Conclusão: Uma proporção significativa dos pacientes apresentava quadro motor assimétrico ou segmentar e reflexos miotáticos preservados. Os resultados obtidos delineiam aspectos clínicos atípicos na SGB em crianças e podem ajudar na definição diagnóstica e instituição de tratamento precoce. Palavras-chave: 1. Síndrome de Guillain-Barré 2. Síndrome de Miller Fisher 3. Assimetria 4. Epidemiologia 


\begin{abstract}
SAMPAIO, PHMA. Neurological asymmetries in children with Guillain-Barré syndrome. 2017. 62f. Dissertação de mestrado. Faculdade de Medicina de Ribeirão Preto, Universidade de São Paulo, São Paulo, 2017.
\end{abstract}

Guillain-Barré syndrome (GBS) is an acute, inflammatory, peripheral neuropathy that has been being defined as an ascending flaccid tetraparesis. Atypical presentations can be frequent, particularly in children, leading to greater challenges in the diagnosis. Objectives: To analyze the epidemiological data and the prevalence of motor asymmetries in the neurological examination of children with GBS. Methods: A total of 40 medical records were analyzed, of children aged 0 to 15 years old diagnosed with GBS, admitted from January 2000 to August 2016. We evaluated the presence of motor asymmetries at the hospital admission, the clinical outcomes and the demographic and clinic-laboratorial characteristics. Results: Two patients had motor asymmetries at hospital admission and three patients admitted with symmetric tetraparesis had an initial motor asymmetry before admission. One patient progressed to asymmetric tetraparesis after being initially admitted with symmetric weakness. Eight other cases had segmental weakness at admission. Motor asymmetry and segmental weakness correlated with a static progression of symptoms $(\mathrm{p}=0.004)$ and these patients tended to be younger, but this difference was not significant $(\mathrm{p}=0.08)$. Eleven patients had preserved deep tendon reflexes and one exhibited hyperreflexia at the hospital admission. Most patients were admitted on wheel-chair or bedridden, and at discharge the majority could walk with or without help. Five children required mechanical ventilation and no patient died. Conclusion: A significant proportion of patients had asymmetric or segmental weakness and preserved deep tendon reflexes. Those results show that the so-called atypical clinical findings in children with GBS are not uncommon, and needs to be kept in mind to allow an earlier diagnosis and treatment.

Keywords: 1. Guillain-Barré syndrome 2. Miller Fisher syndrome 3. Asymmetry 4. Epidemiology 


\section{LISTA DE TABELAS}

Tabela 1. Critérios diagnósticos da Síndrome de Guillain-Barré

Tabela 2. Achados neurológicos assimétricos, segmentares e progressão do quadro motor

Tabela 3. Comparação dos pacientes com quadro assimétrico e segmentar àqueles com tetraparesia simétrica na admissão hospitalar

Tabela 4. Eventos precedendo o início dos sintomas

Tabela 5. Primeiros sintomas observados pelo paciente ou parente

Tabela 6. Achados de exame neurológico na admissão hospitalar 


\section{LISTA DE GRÁFICOS}

Gráfico 1. Número de casos por mês de admissão hospitalar

Gráfico 2. Número de casos por variante diagnóstica da Síndrome de Guillain-Barré

Gráfico 3. Escala funcional de Hughes na admissão, no nadir e na alta hospitalar 


\section{LISTA DE SIGLAS}
AMAN
Acute motor axonal neuropathy
AMSAN
Acute motor and sensory axonal neuropathy
CID-10
Classificação Internacional de Doenças. $10^{\mathrm{a}}$ revisão.
DRS
Departamento Regional de Saúde
EB
Encefalite de Bickerstaff
GM1
Gangliosídeo-tetraexosilmonosialosídeo
IgIV
Imunoglobulina humana em altas doses por via intravenosa
IIQ
Intervalo interquartil
LCR
Líquido cefalorraquidiano
MRC
Medical Research Council
OMS
Organização Mundial da Saúde
PAMC
Potencial de ação muscular composto
PAS
Potencial de ação sensitivo
PBCF
Paralisia bráquio-cérvico-faríngea
PIDA
Polirradiculoneuropatia inflamatória desmielinizante aguda
$\mathrm{PNC}$
Polineurite craniana
$\mathrm{RM}$
Ressonância magnética
SGB
Síndrome de Guillain-Barré
SGB-P
Síndrome de Guillain-Barré paraparético
SMF
Síndrome de Miller Fisher 


\section{SUMÁRIO}

1. INTRODUÇÃO

2. OBJETIVOS

2.1. GERAIS

2.2. ESPECÍFICOS

3. PACIENTES E MÉTODOS

3.1. SELEÇÃO DE PACIENTES

3.2. CRITÉRIOS DE INCLUSÃO

3.3. CRITÉRIOS DE EXCLUSÃO 20

3.4. VARIÁVEIS 20

3.5. COLETA DE DADOS 22

3.6. ESCORE SOMADO DA MEDICAL RESEARCH COUNCIL, ESCALA

FUNCIONAL DE HUGHES E DESFECHOS MOTORES 22

4. ANÁLISE ESTATÍSTICA

5. RESULTADOS

5.1. ACHADOS NEUROLÓGICOS ASSIMÉTRICOS, SEGMENTARES

E PROGRESSÃO DO QUADRO MOTOR

5.2. EVENTOS PRECEDENTES

5.3. SINTOMAS INICIAIS $\quad 30$

5.4. ACHADOS DO EXAME NEUROLÓGICO INICIAL 31

5.5. NADIR NEUROLÓGICO 33

5.6. EXAMES COMPLEMENTARES

5.7. DIAGNÓSTICO, TRATAMENTO E DESFECHO 35

$\begin{array}{ll}\text { 6. DISCUSSÃO } & 38\end{array}$

7. CONCLUSÃO 43

REFERÊNCIAS $\quad 44$

$\begin{array}{ll}\text { ANEXOS } & 48\end{array}$

ANEXO 1 - FORMULÁRIO DE PESQUISA 48

ANEXO 2 - ESCORE SOMADO DA MEDICAL RESEARCH COUNCIL

ANEXO 3 - ESCALA FUNCIONAL DE HUGHES 58

ANEXO 4 - CASOS ILUSTRATIVOS DE ASSIMETRIA

ANEXO 5 - APROVAÇÃO DO COMITÊ DE ÉTICA 62 


\section{INTRODUÇÃO}

A Síndrome de Guillain-Barré (SGB), descrita em 1916 por Guillain, Barré e Strohl, é uma neuropatia periférica inflamatória de instalação aguda e apresentação clínica variada. Em sua forma mais comum, caracteriza-se pela progressão aguda de fraqueza simétrica e ascendente, associada à ausência de reflexos tendíneos profundos. $\mathrm{O}$ curso da doença é monofásico, geralmente pós-infeccioso e responsivo à imunoterapias (KORINTHENBERG, 2013).

$\mathrm{Na}$ era pós-poliomielite, a SGB tornou-se a principal causa de paralisia flácida aguda em crianças (JONES, 2000). A SGB pode afetar indivíduos de qualquer faixa etária, embora a incidência aumente linearmente com a idade. A SGB tem uma incidência anual que varia de 1 a 2 casos por 100.000 habitantes por ano. Em pacientes com até 15 anos, a incidência é menor, variando de 0,39 a 0,63 casos por 100.000 habitantes, conforme um estudo epidemiológico brasileiro (DIAS-TOSTA e KÜCKELHAUS, 2002). Homens são afetados 1,5 mais vezes que mulheres em todas as faixas etárias (SEJVAR et al., 2011).

A SGB é considerada uma doença autoimune, resultante da ativação de linfócitos T e da produção de anticorpos contra proteínas presentes nos nervos periféricos (HAHN, 1998). Em cerca de dois terços dos casos, os sintomas neurológicos se iniciam entre duas a quatro semanas após um quadro infeccioso, sendo mais comumente precedida por Campylobacter jejuni, Mycoplasma pneumoniae, citomegalovírus e vírus de Epstein-Barr. A SGB pode, também, ser precedida por outros tipos de eventos, tais como imunizações ou cirurgias. Esses eventos corresponderiam ao gatilho para uma resposta autoimune cruzada. Na maioria dos casos, o padrão eletrofisiológico da SGB é o de uma polirradiculoneuropatia desmielinizante (HICKS et al., 2010) e, em cerca de um terço dos pacientes, o padrão eletrofisiológico é indicativo de comprometimento axonal primário. Para esses casos, quando a infecção precedente é devida à bactéria Campylobacter jejuni, foi demonstrado um mimetismo molecular entre antígenos de superfície da bactéria e o gangliosídeo-tetraexosilmonosialosídeo (GM1). Este gangliosídeo é um dos componentes do axolema (YUKI et al., 2004). 
A SGB é heterogênea com diversas manifestações e variantes clínicas. A polirradiculoneuropatia inflamatória desmielinizante aguda (PIDA) é a forma de apresentação clínica mais frequente da síndrome (YUKI e HARTUNG, 2012). Sintomas sensitivos positivos iniciais, como parestesias ou dores em extremidades, costumam preceder o quadro motor de horas a dias. Segue-se a fraqueza muscular aguda e progressiva, relativamente simétrica e ascendente, acompanhada da ausência ou hipoatividade dos reflexos miotáticos. O grau de fraqueza pode variar desde o comprometimento discreto da marcha até à paralisia completa dos músculos apendiculares, respiratórios e bulbares. O sétimo nervo craniano é o mais frequentemente afetado e a diparesia facial é uma manifestação clínica habitual. Aproximadamente metade dos casos evoluem com algum tipo de disfunção do sistema autonômico, como labilidade pressórica, diaforese, alterações pupilares e distúrbios urinários e fecais (HAHN, 1998; KOUL e ALFUTAISI, 2008; LIN et al., 2012; YUKI e HARTUNG, 2012).

Diversas variantes clínicas foram referidas na SGB e recentemente uma nova classificação foi proposta (WAKERLEY et al., 2014). Cada variante tem características clínicas, eletrofisiológicas e evolutivas particulares. Formas mistas, denominadas síndrome de sobreposição, podem ocorrer. A neuropatia axonal motora aguda (AMAN - acute motor axonal neuropathy) é uma forma exclusivamente axonal motora da SGB. Distingue-se da forma mais frequente, a PIDA, pelo comprometimento exclusivamente motor e achados eletrofisiológicos indicativos de uma axonopatia (KORINTHENBERG, 2013). Trata-se de um tipo de comprometimento que ocorre com maior frequência nos países asiáticos e está associada à precedência pela infecção por Campylobacter jejuni (YUKI et al., 2004). A neuropatia axonal sensitivo-motora aguda (AMSAN - acute motor and sensory axonal neuropathy) inclui o comprometimento de fibras nervosas sensitivas, o que se reflete no comprometimento da sensibilidade (GRIFFIN et al., 1995; KALITA et al., 2014). A Síndrome de Miller Fisher (SMF) é composta pela tríade de oftalmoparesia extrínseca, ataxia e arreflexia, podendo vir acompanhada de fraqueza muscular. Quando acompanhada de alteração do nível de consciência e sinais de comprometimento do tronco encefálico, causando uma rombencefalite, é denominada de encefalite de Bickerstaff (EB). Formas focais de 
apresentação, tais como a SGB paraparética (SGB-P), paralisia bráquio-cérvico-faríngea (PBCF) e polineurite craniana (PNC) também são encontradas, ainda que em menor frequência (SHAHRIZAILA e YUKI, 2013; WAKERLEY et al., 2014).

Em 90\% dos casos, o declínio neurológico progride em até quatro semanas. Atingido o nadir, após um período denominado de platô, inicia-se a regressão espontânea, lenta e gradual, dos déficits neurológicos, ao longo de semanas a meses. Após esse período, pode-se constatar o retorno completo à normalidade clíniconeurológica ou haver evolução para um quadro sequelar (HAHN, 1998). O curso clínico em crianças costuma ser mais benigno que em adultos, com uma menor duração dos sintomas e de complicações. Inclui-se entre estas a necessidade menos frequente de intubação orotraqueal e de ventilação mecânica (KOUL e ALFUTAISI, 2008; LEE et al., 2008).

Não há exame complementar confirmatório ou excludente do diagnóstico de SGB, especialmente no início do quadro. O diagnóstico á baseado em achados da história e exame físico, punção lombar e estudos eletrofisiológicos (ASBURY e CORNBLATH, 1990). Os critérios diagnósticos da SGB estão sumarizados na Tabela 1. Outros critérios diagnósticos existem, como os critérios de Brighton, mas seu uso é orientado para pesquisa uma vez que exclui a maioria das variantes da SGB (FOKKE, C. et al). Em pacientes com SGB, o exame do líquido cefalorraquidiano (LCR) por punção lombar pode revelar o aumento de proteínas (acima de 50mg/dl) associado à contagem de leucócitos normal (menos que 10 leucócitos por $\mathrm{mm}^{3}$ ), achado conhecido como dissociação albuminocitológica (YUKI e HARTUNG, 2012; WAKERLEY et al., 2014; WILLISON et al., 2016). Entretanto, até metade dos pacientes pode ter o exame do LCR normal na primeira semana de evolução. Os estudos eletrofisiológicos são importantes para a confirmação do diagnóstico e para a classificação entre os subtipos desmielinizantes e axonais (WILLISON et al., 2016). Achados eletroneuromiográficos sugestivos de desmielinização incluem a redução da velocidade de condução motora e sensitiva, aumento das latências motoras distais, bloqueio parcial da condução motora, dispersão temporal e aumento da latência das ondas F. Achados que sugerem as formas axonais incluem a redução da amplitude dos potenciais motores e ou sensitivos e bloqueio parcial da condução motora transitório (também chamado de bloqueio 
reversível) (UNCINI e KUWABARA, 2012; CHANSON e ECHANIZ-LAGUNA, 2014). Assim como no exame de LCR, os achados eletroneuromiográficos podem ser normais na primeira semana de evolução. A ressonância magnética (RM) pode evidenciar a captação de contraste nas eminências das raízes nervosas, na cauda equina e nos nervos cranianos (MULKEY et al., 2010; YIKILMAZ et al., 2010; ZUCCOLI et al., 2011; YUKI e HARTUNG, 2012).

\section{Tabela 1. Critérios diagnósticos da Síndrome de Guillain-Barré ${ }^{1}$}

Progressão de fraqueza apendicular essencialmente simétrica, pode começar somente nas pernas.

Envolvimento de nervos cranianos

Ausência ou redução dos reflexos miotáticos dentro da primeira semana do quadro

Instabilidade do sistema nervoso autonômico

Dor cervical, dorsal ou em extremidades, geralmente mal localizada

Progressão das alterações ao longo de dias até quatro semanas

Parestesias em mãos e pés

Achados contrários ao diagnóstico de SGB

Fraqueza assimétrica persistente

Presença de nível sensitivo

Disfunção urinária ou fecal proeminente

Mais de 50 leucócitos $/ \mathrm{mm}^{3}$ no LCR

Presença de células polimorfonucleares no LCR

\section{Anormalidades laboratoriais que reforçam o diagnóstico de SGB}

Contagem de proteínas no LCR $>45 \mathrm{mg} / \mathrm{dl}$ dentro de 3 semanas do início dos sintomas Achados neurofisiológicos consistentes com uma polineuropatia inflamatória aguda em pelo menos dois membros:

1. Redução da velocidade de condução motora ou sensitiva ( $<80 \%$ do limite inferior da normalidade para idade)

2. Bloqueio de condução ou dispersão temporal do potencial de ação muscular composto (PAMC)

3. Prolongamento das latências distais

4. Anormalidades das ondas F (ausência, impersistência e dispersão)

5. Critérios para as formas axonais incluem a falta de critérios neurofisiológicos de desmielinização, associado à redução das amplitudes do PAMC ou do potencial de ação sensitivo (PAS) ou presença de bloqueio de condução reversível.

1. Adaptado de: ASBURY e CORNBLATH. Assessment of current diagnostic criteria for Guillain-Barré syndrome. Annals of Neurology, 1990.

Os tratamentos para a SGB incluem a administração de imunoglobulina humana intravenosa em altas doses (IgIV) ou a plasmaférese (RAPHAEL et al., 2012; HUGHES 
et al., 2014). Uma pequena parcela dos pacientes pode apresentar flutuações clínicas, definida como uma piora neurológica após um período de estabilidade clínica, que se inicia até 8 semanas após o início do tratamento (FOKKE et al., 2014). Nesses casos, o re-tratamento com a mesma modalidade de tratamento instituída previamente pode ser considerado (RAJABALLY, 2012; GODOY e RABINSTEIN, 2015). Independentemente da instituição de imunoterapias para o tratamento, os pacientes devem ser monitorados atentamente em decorrência do risco de insuficiência respiratória e disautonomia (arritmias, labilidade pressórica). Medidas de suporte em regime de internação em Unidade de Terapia Intensiva para tratamento dos referidos distúrbios são frequentemente necessárias (VAN DOORN, 2013).

$\mathrm{O}$ reconhecimento imediato da SGB em crianças, nem sempre é simples. Tratase de uma população na qual os achados neurológicos podem ser limitados, pelas dificuldades da anamnese e do exame neurológico. Além disso, apresentações atípicas são mais frequentes nessa faixa etária (TANG et al., 2011; KARIMZADEH et al., 2012; LIN et al., 2012; DEVOS et al., 2013; KUMAR et al., 2015). Em consequência, erros e atrasos no diagnóstico da SGB são mais comuns em crianças (ROODBOL et al., 2011). O presente estudo tem por objetivo avaliar retrospectivamente a prevalência de achados clínicos assimétricos da SGB nessa faixa etária. Foram também avaliados outros dados demográficos dessa população, incluindo a prevalência de apresentações clínicas segmentares e focais, assim como o perfil das diferentes variantes da SGB, seus desfechos clínicos e complicações. 


\section{OBJETIVOS}

\subsection{GERAIS}

Descrever a apresentação clínica inicial, a prevalência de achados clínicos assimétricos e os desfechos clínicos da SGB em crianças.

\subsection{ESPECÍFICOS}

- Determinar a prevalência de apresentação clínica assimétrica na SGB em crianças.

- Verificar o tempo médio de generalização dos achados assimétricos e a forma de progressão.

- Referir os tratamentos utilizados e sua correlação com os desfechos clínicos.

- Descrever as características demográficas e clinico-laboratoriais desse grupo, incluindo as diferentes variantes da SGB, o perfil de achados clínicos na admissão e no nadir, as complicações clínicas e os resultados de exames complementares. 


\section{PACIENTES E MÉTODOS}

\subsection{SELEÇÃO DE PACIENTES}

A seleção de pacientes foi realizada através da revisão de bancos de dados e prontuários médicos de pacientes seguidos no serviço de Neurologia do Hospital das Clínicas da Faculdade de Medicina de Ribeirão Preto, Universidade de São Paulo (HCFMRP-USP), no período de janeiro de 2000 a dezembro de 2016, que tenham apresentado o Código G61.0 da Classificação Estatística Internacional de Doenças e Problemas Relacionados com a Saúde 10ª revisão (CID-10) da Organização Mundial da Saúde (OMS), correspondente ao diagnóstico de Síndrome de Guillain-Barré.

Foram inclusos pacientes com até 15 anos completos à época do início da doença que tenham sido avaliados no serviço de Neurologia do HCFRMP-USP durante a fase aguda, antes do período de regressão dos sintomas neurológicos. Os pacientes deveriam ter o diagnóstico clínico de SGB firmado em dados clínicos e laboratoriais consistentes, de acordo com os critérios clínicos vigentes (ASBURY e CORNBLATH, 1990; UNCINI e KUWABARA, 2012; WAKERLEY et al., 2014) - vide Tabela 1.

Todos os pacientes foram submetidos a pelo menos um exame eletroneuromiográfico, a fim de se excluir outras neuropatias que possam mimetizar a SGB, assim como satisfazer os critérios diagnósticos (FOKKE et al., 2014; WAKERLEY et al., 2014). Foram incluídas tanto as formas desmielinizantes, quanto as axonais da SGB, assim como a SMF e variantes, de acordo com a classificação mais recente (WAKERLEY et al., 2014).

\subsection{CRITÉRIOS DE INCLUSÃO}

Para serem incluídos neste estudo, os indivíduos selecionados tiveram que preencher os seguintes critérios:

- Ter até 15 anos completos à época do início da doença. 
- Ter o diagnóstico firmado de SGB ou SMF, incluindo as variantes clínicas. Esse diagnóstico deve ser baseado nos achados clínicos e apoiado em exames complementares.

- Conter dados pessoais, da história e evolução clínica completos, de forma clara, nos prontuários médicos.

- Ter sido submetido à avaliação clínica e tratamento pela equipe de Neurologia do HCFMRP-USP a partir da entrada no hospital e do diagnóstico na fase aguda da doença, bem como ao longo seguimento neurológico posterior.

\subsection{CRITÉRIOS DE EXCLUSÃO}

- Pacientes que não foram submetidos a estudos eletrofisiológicos do serviço de Neurofisiologia Clínica do HCFMRP-USP.

\subsection{VARIÁVEIS}

Para cada paciente, foram analisadas as seguintes variáveis:

- Idade, em anos completos, no início do quadro neurológico.

- Sexo.

- Data de admissão no HCFMRP-USP.

- Presença de sinais motores assimétricos ou segmentares na admissão hospitalar.

- Progressão para sinais motores simétricos ou generalizados, se inicialmente assimétrico ou segmentar e o número de dias para a progressão dos sintomas.

- Progressão dos sintomas neurológicos no eixo craniocaudal (ascendente, descendente ou estático).

- Presença de evento infeccioso, vacinal ou cirúrgico precedendo o início dos sintomas e o número de dias que precede.

- Primeiro sintoma percebido pelo paciente ou cuidador. 
- Número de dias entre o início dos sintomas neurológicos e sinais motores e a primeira avaliação clínica no HCFMRP-USP.

- Escore da somatória dos graus de força muscular do Medical Research Council (MRC) na admissão e no nadir, incluindo os escores parciais para os lados esquerdo e direito.

- Classificação da motricidade apendicular na admissão hospitalar e no nadir neurológico.

- Reflexos miotáticos na admissão hospitalar e no nadir neurológico.

- Alterações de sensibilidade vibratória e térmico-dolorosa na admissão, incluindo dor, parestesias dolorosas e sinais meníngeos na admissão hospitalar.

- Presença de ataxia sensitiva e/ou cerebelar, alterações de nervos cranianos, disfunção esfincteriana, disautonomia ou alteração do nível de consciência na admissão hospitalar.

- Número de dias entre o início dos sintomas motores e o nadir neurológico.

- Número de dias entre a admissão hospitalar e o nadir neurológico e o número total de dias de platô neurológico.

- Resultado do exame de líquido cefalorraquidiano, incluindo a presença de dissociação albuminocitológica.

- Resultado da ressonância magnética de encéfalo e coluna, se realizado.

- Resultado do exame eletroneuromiográfico.

- Variante da SGB.

- Tratamento utilizado e número de dias entre a data de internação hospitalar e a instituição do tratamento.

- Presença de flutuações neurológica após o tratamento inicial e necessidade de novo tratamento.

- Necessidade de intubação orotraqueal e ventilação mecânica durante a evolução clínica.

- Número de dias de internação hospitalar.

- Escala funcional de Hughes na admissão hospitalar, no nadir neurológico e na alta hospitalar 


\subsection{COLETA DE DADOS}

A extração de dados dos prontuários médicos foi realizada pelo pós-graduando, utilizando um questionário especificamente desenhado para este estudo (Anexo 1). Para variáveis que dependiam da história clínica, foi utilizado como fonte primária de informação o primeiro registro médico do paciente (primeiro atendimento ambulatorial, caso novo ou ficha de atendimento da Unidade de Emergência do HCFMRP-USP).

No caso de variáveis que dependiam de achados de exame físico do paciente, foi utilizado como fonte primária de informação o primeiro registro de exame neurológico do paciente realizado por algum membro da equipe de neurologia do HC-FMRP-USP, preferencialmente aquela contida no primeiro atendimento ambulatorial, caso novo ou ficha de atendimento da Unidade de Emergência do HCFMRP-USP. Outros sinais clínicos na admissão que estivessem descritos em avaliações neurológicas subsequentes (por exemplo, após a reavaliação do caso com o preceptor disponível na época) foram considerados, contanto que tivessem ocorrido com até 24 horas da avaliação inicial. No caso de dados divergentes da avaliação inicial, foram considerados aqueles obtidos pelo membro mais graduado da equipe.

\subsection{ESCORE SOMADO DA MEDICAL RESEARCH COUNCIL, ESCALA FUNCIONAL DE HUGHES E DESFECHOS MOTORES}

O escore somado da Medical Research Council (MRC) (Anexo 2) tem por objetivo capturar o grau de força global de pacientes e se obtém somando-se os valores de força muscular dos seguintes grupos musculares: abdução dos membros superiores, flexão dos cotovelos, extensão dos punhos, flexão dos quadris, extensão dos joelhos e dorsiflexão dos pés, conforme a graduação da MRC (graduado de 0 a 5). O escore compreende valores entre 0 (paralisia completa) a 60 (força normal) (KLEYWEG et al., 1991).

Foram anotados os escores parciais para o lado direito e esquerdo, compreendendo 0 a 30 pontos cada um. O grau de comprometimento motor foi considerado assimétrico quando houve uma diferença de mais de 5 pontos entre os 
escores de cada lado. No caso de pacientes pouco colaborativos em que o preenchimento do escore somado da MRC não é possível (como crianças pequenas), a fraqueza foi considerada assimétrica somente quando houvesse uma menção clara de assimetria no exame motor descritivo.

A escala funcional de Hughes (Anexo 3) é uma escala que mede os diversos graus de comprometimento funcional ocasionado pela SGB (HUGHES et al., 1992). É medida de $0 \mathrm{a} 6$ pontos, sendo 0 ponto equivalente à ausência completa de alterações funcionais; um ponto equivalente a disfunção leve, sendo o paciente capaz de exercer trabalhos manuais ou correr; dois pontos equivalente à dificuldade de deambulação, capaz de andar ao menos cinco metros sem apoio, porém incapaz de exercer trabalhos manuais ou correr; três pontos equivalente à capacidade de deambulação com apoio por ao menos cinco metros; quatro pontos equivalente à restrição ao leito ou cadeira de rodas; cinco pontos equivalente à necessidade de ventilação mecânica; seis pontos equivalente ao paciente que evoluiu para óbito.

O nadir neurológico foi definido como o maior valor de escala funcional de Hughes ( 0 a 6$)$ ou o menor escore somado MRC $(0$ - 60) atingido pelo paciente, excluindo-se flutuações de até quatro pontos no escore somado da MRC. O platô neurológico foi considerado como o número de dias a partir do nadir até o início da melhora de pelo menos um ponto na escala funcional de Hughes ou aumento de 5 pontos ou mais no escore somado da MRC. Esses valores foram definidos dessa maneira pois o escore somado da MRC tem uma alta concordância inter-observador, capaz detectar diferenças de 5 pontos ou mais (KLEYWEG et al., 1991), e diferenças menores que cinco pontos poderiam representar variações inter-observador esperadas na aferição de força muscular. 


\section{ANÁLISE ESTATÍSTICA}

Dados relativos a variáveis numéricas estão apresentados através da média, desvio padrão, mediana e intervalo interquartil (IIQ), enquanto que as variáveis categóricas estão sumarizadas na forma de frequências e proporção. Como a frequência de pacientes com clínica assimétrica na admissão hospitalar foi pequena (somente dois pacientes), optou-se por agrupar os casos assimétricos e segmentares para os testes estatísticos, uma vez que estes casos englobam padrões de acometimento motor atípicos na SGB. Foram avaliadas as correlações entre a presença de apresentações assimétricas e segmentares e variáveis como idade, sexo, presença de antecedentes como infecção de vias aéreas e diarreia, o padrão eletroneuromiográfico, a preservação ou não dos reflexos miotáticos na admissão e no nadir, a progressão, a presença de disautonomia, ao achado de dissociação albuminocitológica no exame de LCR, assim como ao número de dias para ser encaminhado ao HCFMRP-USP, número de dias de internação hospitalar, número de dias de platô neurológico e escore médio da escala funcional de Hughes na admissão e no nadir neurológico. Para as variáveis numéricas, foi utilizado o teste $U$ de Mann-Whitney, após o teste de normalidade de Shapiro-Wilk ter descartado a hipótese de distribuição normal nestas variáveis. O teste exato de Fisher foi utilizado para avaliar a associação entre variáveis categóricas. $\mathrm{O}$ erro do tipo I foi fixado a 0,05 em todos os testes de hipóteses. Os resultados significativos foram controlados para idade e sexo, através de regressão logística multivariada. A análise estatística foi realizada através do software STATA para Windows e MacOS. 


\section{RESULTADOS}

Um total de 40 pacientes com SGB admitidos entre janeiro de 2000 e agosto 2016 preencheram os critérios de inclusão para o estudo. Desses, vinte e três $(57,5 \%)$ eram do sexo masculino e dezessete $(42,5 \%)$ do feminino. As idades variaram entre 8 meses e 15 anos (média 7,7 \pm 5 anos). Em relação à procedência dos pacientes, somente dezessete $(42,5 \%)$ pertenciam ao Departamento Regional de Saúde (DRS) XIII (Ribeirão Preto), enquanto o restante dos casos pertencia aos DRS III (Araraquara), V (Barretos), VIII (Franca), IX (Marília), X (Piracicaba) e XV (São José do Rio Preto). Um paciente era proveniente do Paraná e dois eram de Minas Gerais. A maioria das admissões hospitalares se concentraram entre agosto e dezembro, com seis casos em cada. A distribuição dos casos por mês encontra-se sumarizada no Gráfico 1.

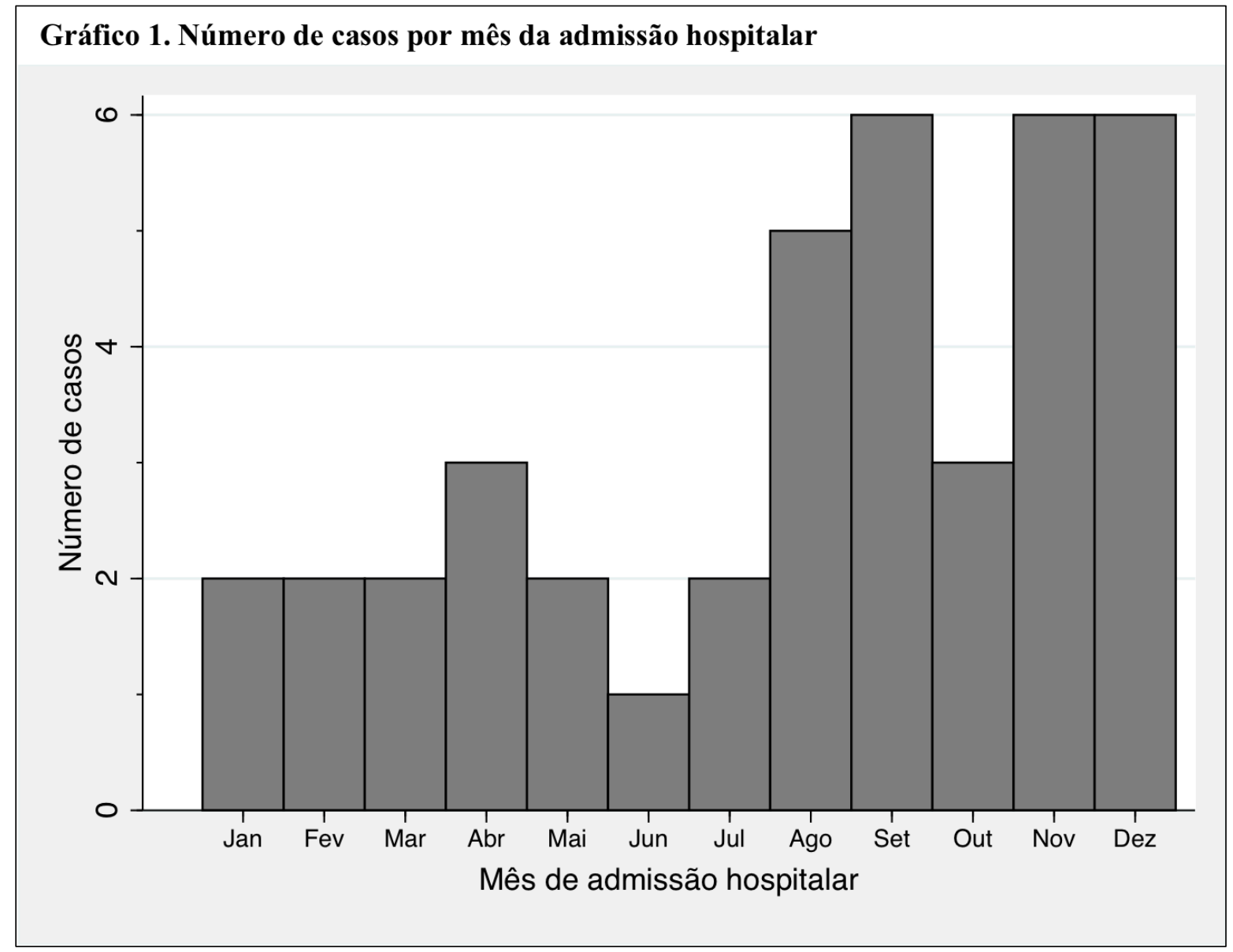




\subsection{ACHADOS NEUROLÓGICOS ASSIMÉTRICOS, SEGMENTARES E PROGRESSÃO DO QUADRO MOTOR}

Seis pacientes $(15 \%)$ apresentaram assimetria motora no exame neurológico em algum momento da evolução clínica. Um paciente foi admitido apresentando uma tetraparesia assimétrica, com maior comprometimento da força muscular no membro superior esquerdo, mantendo a assimetria mesmo durante o nadir (Anexo 4, caso I). Outro paciente foi admitido com paraparesia assimétrica, pior à esquerda, que também se manteve durante o nadir (Anexo 4, caso II). Uma criança que inicialmente se apresentou com uma tetraparesia simétrica evoluiu para uma tetraparesia assimétrica de predomínio no dimidio esquerdo, após cinco dias de internação hospitalar (Anexo 4, caso III). Outros três pacientes apresentaram uma assimetria motora inicial bem documentada na história clínica, mas na admissão hospitalar já exibiam uma tetraparesia simétrica. Um paciente abriu com quadro de fraqueza assimétrica dos membros superiores, que após um dia progrediu de maneira descendente para fraqueza simétrica dos quatro membros (Anexo 4, caso IV) e em dois casos a fraqueza inicialmente observada foi no membro inferior esquerdo (Anexo 4, casos V e VI).

Além dos pacientes com quadro motor assimétrico, outros oito pacientes exibiam um padrão de fraqueza segmentar ou focal na admissão hospitalar. Cinco crianças apresentavam fraqueza simétrica, mas exclusiva dos membros inferiores, sendo que somente uma delas evoluiu para tetraparesia simétrica no nadir. Uma criança apresentava fraqueza exclusivamente proximal com músculos distais poupados e outra iniciou com fraqueza distal de membros superiores que evoluiu com fraqueza dos quatro membros exclusivamente distal. Ambas permaneceram com esse padrão motor mesmo durante o nadir. Um paciente apresentou inicialmente um quadro exclusivo de múltiplos nervos cranianos, sem fraqueza apendicular, progredindo para uma tetraparesia proximal de leve intensidade no nadir, após três dias da internação. Por fim, outros três pacientes foram admitidos com tetraparesia simétrica, mas tinham uma história de acometimento segmentar antes da internação: duas crianças com paraparesia de membros inferiores que se sustentou por dias (uma delas por onze dias) antes de progredir para os quatro membros e uma com paresia membros superiores. 
A progressão da assimetria e do acometimento motor segmentar comportou-se da seguinte maneira: vinte e três pacientes $(57,5 \%)$ cuja manifestação inicial foi tetraparesia simétrica mantiveram-se neste estado durante toda a evolução do quadro. Três pacientes (7,5\%) com clínica assimétrica inicial, eventualmente evoluíram para um quadro motor simétrico antes da admissão hospitalar (Anexo 4, casos IV, V e VI). Como descrito anteriormente, um paciente $(2,5 \%)$ evoluiu de tetraparesia simétrica para assimétrica (Anexo 4, caso III), e dois mantiveram a assimetria durante a evolução (Anexo 4, casos I e II). Dois pacientes (5\%) com quadro motor segmentar progrediram para um quadro de fraqueza generalizada, enquanto que os outros seis pacientes $(15 \%)$ com quadro motor segmentar não evoluíram mesmo no nadir. A progressão dos sintomas motores no eixo craniocaudal foi ascendente em vinte e seis $(65 \%)$ pacientes, descendente em seis (15\%) e sem progressão em oito (20\%). Os dados estão sumarizados na Tabela 2.

Tabela 2. Achados neurológicos assimétricos, segmentares e progressão do quadro motor

\begin{tabular}{lc}
\hline & N (proporção em \%) \\
\hline Quadro motor na admissão hospitalar & $30(75 \%)$ \\
Tetraparesia simétrica & $2(5 \%)$ \\
Fraqueza assimétrica & $8(20 \%)$ \\
Fraqueza segmentar & \\
Progressão da assimetria ${ }^{1}$ & $26(65 \%)$ \\
Início e progressão de tetraparesia simétrica & $2(5 \%)$ \\
Início e progressão assimétricos & $3(7,5 \%)$ \\
Início assimétrico com progressão para simetria & $1(2,5 \%)$ \\
Início simétrico com progressão para assimetria & $6(15 \%)$ \\
Início e progressão segmentar & $2(5 \%)$ \\
Início segmentar com progressão generalizada & \\
Progressão das alterações neurológicas & \\
Ascendente & $26(65 \%)$ \\
Descendente & $6(15 \%)$ \\
Sem progressão & $8(20 \%)$ \\
\hline 1. Total de pacientes: 40 &
\end{tabular}


Juntos, somaram-se um total de dez casos admitidos no HCFMRP-USP com fraqueza assimétrica ou segmentar. A Tabela 3 contém um sumário dos achados referentes a esse grupo em comparação com grupo admitido com tetraparesia simétrica.

Tabela 3. Comparação dos pacientes com quadro assimétrico e segmentar àqueles com tetraparesia simétrica na admissão hospitalar

\begin{tabular}{llccc}
\hline Variável & & Simétrico $(\mathrm{n}=30)$ & $\begin{array}{c}\text { Assimétrico/Segmentar } \\
(\mathrm{n}=10)\end{array}$ & $\mathrm{P}^{1}$ \\
\hline Idade (anos) & Masculino & $8,4 \pm 4,9$ & $5,4 \pm 4,9$ & 0,08 \\
Sexo & $15(50 \%)$ & $8(80 \%)$ & $\mathrm{NS}$ \\
& Feminino & $15(50 \%)$ & $2(20 \%)$ & $\mathrm{NS}$ \\
Diarreia & & $9(30 \%)$ & $1(10 \%)$ & $\mathrm{NS}$ \\
Infeção de vias aéreas & & $12(40 \%)$ & $4(40 \%)$ & $\mathrm{NS}$ \\
Disautonomia & $11(36,7 \%)$ & $2(20 \%)$ & $\mathrm{NS}$ \\
Reflexos preservados & Admissão & $9(30 \%)$ & $3(30 \%)$ & $\mathrm{NS}$ \\
& Nadir & $9(30 \%)$ & $3(30 \%)$ & $\mathrm{NS}$ \\
Sem progressão no eixo & & $3(10 \%)$ & $5(50 \%)$ & 0,004 \\
craniocaudal & & $13(32,5 \%)$ & $4(40 \%)$ & $\mathrm{NS}$ \\
Dissociação no LCR & & $21(70 \%)$ & $5(50 \%)$ & $\mathrm{NS}$ \\
Padrão da ENMG & Desmielinizante & $6(20 \%)$ & $2(20 \%)$ & $\mathrm{NS}$ \\
& Axonal & $3(10 \%)$ & $2(20 \%)$ & $\mathrm{NS}$ \\
$\mathrm{N}^{\mathrm{o}}$ de dias para ser & Indeterminado & & $6 \pm 3,8$ & $\mathrm{NS}$ \\
encaminhado ao HC & & $1,2 \pm 6,2$ & $8,8 \pm 4,5$ & $\mathrm{NS}$ \\
$\mathrm{N}^{\mathrm{o}}$ de dias de internação & & $9,5 \pm 6,7$ & $6,2 \pm 5,4$ & $\mathrm{NS}$ \\
Tempo de platô (dias) & & $3,4 \pm 1,1$ & $2,9 \pm 1,4$ & $\mathrm{NS}$ \\
Escala de Hughes & Admissão & $3,7 \pm 0,95$ & $3,2 \pm 1,1$ & $\mathrm{NS}$ \\
\hline
\end{tabular}

1. Para variáveis categóricas, utilizou-se o teste exato de Fisher. Para variáveis numéricas, foi utilizado o teste U de MannWhitney. NS: Não significante.

ENMG: Eletroneuromiografia. LCR: Líquido cefalorraquidiano. PIDA: Polirradiculoneuropatia Inflamatória Desmielinizante Aguda. AMAN: Neuropatia Axonal Motora Aguda

A presença de assimetria ou acometimento segmentar na admissão não apresentou correlação estatisticamente significativa com o sexo, a presença de eventos precedentes como infecção de vias aéreas superiores ou diarreia, o padrão eletroneuromiográfico ou com outras características clínicas, como a preservação ou não dos reflexos miotáticos na admissão e no nadir, presença de disautonomia ou achado de dissociação albuminocitológica no exame de LCR. De modo similar, não foi observado diferença significativa entre os dois grupos, em relação ao número de dias para ser encaminhado ao HCFMRP-USP, número de dias de internação hospitalar, número de 
dias de platô neurológico, e escore médio da escala funcional de Hughes na admissão e no nadir neurológico. Notou-se uma tendência de pacientes com assimetria ou acometimento segmentar na admissão serem mais jovens, mas esse achado não teve significância $(\mathrm{p}=0,08)$. Entretanto, observou-se uma associação significante entre a presença de assimetria ou acometimento segmentar na admissão e não progressão dos sintomas motores no eixo craniocaudal $(\mathrm{p}=0,004)$, achado compatível com o fato de que aproximadamente metade dos pacientes com sintomas assimétricos ou segmentares não apresentou generalização dos déficits motores. Esse resultado se manteve mesmo após ajuste para sexo e idade através de regressão logística multivariada.

\subsection{EVENTOS PRECEDENTES}

Trinta e três $(82,5 \%)$ pacientes apresentaram algum tipo de evento antecedendo o início do quadro. Os eventos precedentes estão sumarizados na Tabela 4. O evento pregresso mais comum foi infecção de vias aéreas (16 casos, 40\%), seguido de diarreia (10 casos, 25\%), vacinação (4 casos, 10\%) e evento febril inespecífico (2 casos, $5 \%$ ). Dentre os pacientes com antecedente de vacinação, dois haviam sido vacinados para poliomielite, um deles dezesseis dias antes do início dos sintomas e o outro vinte e cinco dias antes. Ambos tiveram o diagnóstico de PIDA, com achados eletrofisiológicos de desmielinização. Um paciente foi vacinado para o vírus H1N1 trinta e dois dias antes e o último paciente havia sido vacinado para pneumococo (vacina pneumocócica 10valente) onze dias antes. Outros eventos incluíram otite média aguda, varicela, exantema febril, exantema cutâneo inespecífico e picada de carrapato. Este último tinha sinais de desmielinização na ENMG e apresentou quadro de tetraparesia arreflexa associado a rebaixamento do nível de consciência e oftalmoparesia, compatível com diagnóstico de encefaloneurite. À época, em 2007, não foram colhidas sorologias para doença de Lyme. Em sete casos, mais de um evento pregresso foi observado. Em média, os eventos precederam o início do quadro motor em 13,7 \pm 12,7 dias (mediana 10 dias; IIQ 5-18,5 dias). 
Tabela 4. Eventos precedendo o início dos sintomas

\begin{tabular}{|c|c|c|}
\hline & \multicolumn{2}{|c|}{$\mathrm{N}$ (proporção em \%) } \\
\hline Sem evento precedente & \multicolumn{2}{|c|}{$7(17,5 \%)$} \\
\hline Evento precedente $^{2}$ & \multicolumn{2}{|c|}{$33(82,5 \%)$} \\
\hline Infecção de vias aéreas & \multicolumn{2}{|c|}{$16(40 \%)$} \\
\hline Diarreia & \multicolumn{2}{|c|}{$10(25 \%)$} \\
\hline Vacinação & \multicolumn{2}{|c|}{$4(10 \%)$} \\
\hline Evento febril inespecífico & \multicolumn{2}{|c|}{$2(5 \%)$} \\
\hline \multirow[t]{2}{*}{ Outros $^{4}$} & \multicolumn{2}{|c|}{$5(12,5 \%)$} \\
\hline & Média $( \pm \mathrm{DP})$ & Mediana (IIQ) \\
\hline Tempo entre evento e início do quadro (dias) & $13,7( \pm 12,7)$ & $10(5-18,5)$ \\
\hline $\begin{array}{l}\text { IIQ: intervalo interquartil. DP: desvio padrão. } \\
\text { 1. Total de pacientes: } 40 . \\
\text { 2. Foram considerados os eventos precedendo o início } \\
\text { 3. Evento mononucleose-símile: consiste em sintomas } \\
\text { 4. Outros: otite média aguda, varicela, exantema febril, }\end{array}$ & $\begin{array}{l}60 \text { dias. } \\
\text { e e linfadenopatia } \\
\text { inespecífico e pica }\end{array}$ & anato \\
\hline
\end{tabular}

\subsection{SINTOMAS INICIAIS}

Os sintomas iniciais percebidos pelo paciente ou cuidador estão resumidos na Tabela 5. O sintoma com maior prevalência foi a presença de fraqueza apendicular em vinte e quatro casos (60\%), seguido pela dificuldade de marcha (22 casos, $55 \%$ ) e dor (21 casos, 52,5\%). Cefaleia estava presente em seis pacientes (15\%). Alteração urinária ou fecal, dormência e presença de desequilíbrio ou incoordenação foram menos frequentes e observados somente em $2,5 \%$ dos casos. O tempo entre o início dos sintomas até a primeira avaliação no HCFMRP-USP variou de um a vinte e sete dias. Em média, o período de tempo foi de 6,9 \pm 5,7 dias (mediana 6 dias; IIQ 3-9,5 dias). 


\begin{tabular}{lc}
\hline & $N\left(\right.$ proporção em \%) ${ }^{1}$ \\
\hline Fraqueza apendicular & $24(60 \%)$ \\
Dificuldade de marcha & $22(55 \%)$ \\
Dor & $21(52,5 \%)$ \\
Cefaleia & $6(15 \%)$ \\
Alteração em nervos cranianos & $4(10 \%)$ \\
Alteração esfincteriana (urinária/fecal) & $1(2,5 \%)$ \\
Desequilíbrio e/ou incoordenação & $1(2,5 \%)$ \\
Dormência e/ou hipoestesia & $1(2,5 \%)$ \\
\hline
\end{tabular}

1. Total de pacientes: 40 .

\subsection{ACHADOS DO EXAME NEUROLÓGICO INICIAL}

Os dados relativos aos achados de exame neurológico da primeira avaliação no serviço de neurologia do HCFMRP-USP estão sumarizados na Tabela 6. Na admissão hospitalar, as alterações de motricidade apendicular apresentaram-se, em sua maioria (29 casos, 72,5\%), como tetraparesia simétrica. Seis pacientes (15\%) foram admitidos com paraparesia, sendo um deles uma paraparesia assimétrica (Anexo 4, caso II). Observou-se um caso de tetraparesia assimétrica (Anexo 4, caso I), um de fraqueza proximal e um de fraqueza distal. Somente dois pacientes foram admitidos sem fraqueza apendicular. Os reflexos miotáticos estavam abolidos ou diminuídos em vinte e oito pacientes $(70 \%)$, preservados em onze pacientes $(27,5 \%)$ e uma criança apresentava hiperreflexia.

Uma pequena proporção de pacientes apresentava alterações da sensibilidade no exame físico da admissão. Três pacientes exibiam hipoestesia vibratória, um paciente exibia hipoestesia térmico-dolorosa e um paciente apresentava alterações sensitivas tanto para sensibilidade vibratória quanto térmico-dolorosa. A maioria dos pacientes (27 casos, 67,5\%) tinha o exame de sensibilidade normal, enquanto que em oito casos $(20 \%)$ não foi possível determinar o grau de comprometimento sensitivo devida à não colaboração dos pacientes. Similarmente, somente cinco pacientes (12,5\%) exibiam ataxia cerebelar e um paciente apresentava ataxia mista (cerebelar e sensitiva) na admissão. Nenhum paciente apresentou exclusivamente ataxia sensitiva. 


\begin{tabular}{|c|c|}
\hline & $\mathrm{N}$ (proporção em \%) ${ }^{1}$ \\
\hline \multicolumn{2}{|l|}{ Motricidade apendicular } \\
\hline Tetraparesia simétrica & $29(72,5 \%)$ \\
\hline Paraparesia & $6(15 \%)$ \\
\hline Tetraparesia assimétrica & $1(2,5 \%)$ \\
\hline Fraqueza proximal & $1(2,5 \%)$ \\
\hline Fraqueza distal & $1(2,5 \%)$ \\
\hline Sem fraqueza apendicular & $2(5 \%)$ \\
\hline \multicolumn{2}{|l|}{ Reflexos miotáticos } \\
\hline Arreflexia ou hiporreflexia & $28(70 \%)$ \\
\hline Normorreflexia & $11(27,5 \%)$ \\
\hline Hiperreflexia & $1(2,5 \%)$ \\
\hline \multicolumn{2}{|l|}{ Exame de sensibilidade } \\
\hline Normal & $27(67,5 \%)$ \\
\hline Hipoestesia vibratória & $3(7,5 \%)$ \\
\hline Hipoestesia térmico-dolorosa & $1(2,5 \%)$ \\
\hline Hipoestesia mista & $1(2,5 \%)$ \\
\hline Não colaborativo & $8(20 \%)$ \\
\hline \multicolumn{2}{|l|}{ Coordenação } \\
\hline Normal & $34(85 \%)$ \\
\hline Ataxia cerebelar & $5(12,5 \%)$ \\
\hline Ataxia mista & $1(2,5 \%)$ \\
\hline Ataxia sensitiva & 0 \\
\hline Dor & $17(42,5 \%)$ \\
\hline Paralisia facial & $13(32,5 \%)$ \\
\hline Fraqueza bulbar ${ }^{2}$ & $7(17,5 \%)$ \\
\hline Nervos III, IV, VI $^{3}$ & $7(17,5 \%)$ \\
\hline Disautonomia & $6(15 \%)$ \\
\hline Alteração da consciência & $4(10 \%)$ \\
\hline Disfunção esfincteriana (urinária/fecal) & $4(10 \%)$ \\
\hline Meningismo & $3(7,5 \%)$ \\
\hline
\end{tabular}

1. Total de pacientes: 40 .

2. Fraqueza dos nervos IX, X, XI, XII.

3. Inclui as várias apresentações de oftalmoparesia e ptose palpebral.

Dor foi um sintoma frequente, em dezessete casos $(42,5 \%)$ e foi a queixa predominante em três pacientes. Entres os nervos cranianos, o nervo facial foi o mais frequentemente acometido, com treze pacientes $(32,5 \%)$ apresentando um padrão de paralisia facial periférica. Sete pacientes $(17,5 \%)$ exibiam fraqueza bulbar 
(acometimento dos nervos cranianos IX, X, XI e XII). A fraqueza dos nervos responsáveis pela motricidade ocular (nervos cranianos III, IV e VI) também estava presente em sete casos $(17,5 \%)$. Outros achados menos comuns incluíam a presença de disautonomia em seis casos (15\%), a alteração do nível de consciência em quatro pacientes (10\%), alterações esfincterianas (urinária/fecal) em quatro pacientes $(10 \%)$ e a presença de meningismo em três pacientes $(7,5 \%)$.

Dados relativos ao escore somado da MRC estavam disponíveis para dezoito pacientes (45\%). Em média, o escore na admissão hospitalar foi de 39,8 \pm 11,5 pontos (mediana 42 pontos, IIQ 32-48 pontos).

\subsection{NADIR NEUROLÓGICO}

Dados relativos à motricidade e aos reflexos miotáticos no nadir neurológico estão delineados a seguir. A maioria dos casos (28 casos, 70\%) já se encontrava no nadir neurológico à admissão hospitalar. Consequentemente, os achados no nadir foram semelhantes aos da admissão. Trinta e um (77,5\%) pacientes exibiam uma tetraparesia simétrica no nadir neurológico, cinco (12,5\%) estavam paraparéticos e dois $(5 \%)$ apresentavam uma tetraparesia assimétrica. Fraqueza proximal e fraqueza distal ocorreram em um paciente $(2,5 \%)$ cada. A maioria (28 casos, $70 \%$ ) dos pacientes estava com os reflexos miotáticos reduzidos ou abolidos, dez pacientes (25\%) ainda apresentavam reflexos miotáticos preservados e dois pacientes (5\%) apresentavam hiperreflexia. O tempo médio entre o início dos sintomas e o nadir neurológico foi de 6,8 \pm 5,4 dias (mediana 6 dias, IIQ 3-8 dias), enquanto que o período médio de duração do platô neurológico foi de 8,9 \pm 6,6 dias (mediana 6 dias, IIQ 4-13 dias).

\subsection{EXAMES COMPLEMENTARES}

O resultado do exame de LCR estava disponível para trinta e seis dos quarenta pacientes. A média de contagem de células no LCR foi de 4,6 $\pm 6,8$ leucócitos por $\mathrm{mm}^{3}$ (mediana 2 leucócitos $/ \mathrm{mm}^{3}$, IIQ 1 - 3,3 leucócitos $/ \mathrm{mm}^{3}$ ). Trinta e um pacientes 
apresentavam uma contagem de leucócitos $<10 / \mathrm{mm}^{3}$, enquanto cinco apresentavam uma contagem entre 10 e $50 / \mathrm{mm}^{3}$ leucócitos. Nenhum paciente apresentou contagem de células maior do que $50 / \mathrm{mm}^{3}$. Em média, a contagem de proteínas no LCR foi de 80,8 $\pm 80,7 \mathrm{mg} / \mathrm{dl}$ (mediana $51,5 \mathrm{mg} / \mathrm{dl}$, IIQ 32,99- $97 \mathrm{mg} / \mathrm{dl}$ ). A coleta do LCR foi realizada em média com 5,9 \pm 4,9 dias do início do quadro. Dos trinta e seis resultados de LCR colhidos, dezessete $(47,2 \%)$ preenchiam os critérios para dissociação albuminocitológica (contagem de leucócitos $<10 / \mathrm{mm}^{3}$ e dosagem de proteínas $>50$ $\mathrm{mg} / \mathrm{dl}$ ). Em seis casos, o LCR inicial não demonstrava dissociação albuminocitológica, sendo repetido em um segundo tempo. Desses, cinco apresentaram a conversão do LCR para o padrão dissociado.

$\mathrm{O}$ exame de RM da coluna foi realizado em cinco pacientes, sendo solicitado em quatro casos por conta de apresentações atípicas (fraqueza assimétrica descendente, tetraparesia assimétrica, paraparesia e polineurite craniana) e em uma após falta de resposta inicial à terapia com IgIV. Dos cinco pacientes, dois demonstraram captação radicular de contraste e os três restantes tinham RM da coluna normal. Nove pacientes realizaram o exame de RM de encéfalo. Os exames foram solicitados para diagnóstico diferencial, em pacientes com acometimento da motricidade ocular extrínseca ou ataxia, a fim de se excluir lesões no sistema nervoso central. Um paciente apresentava focos de alteração de substância branca, sugerindo gliose ou alteração da mielina, outro apresentava atraso global e simétrico da mielinização, e um terceiro apresentava hipersinal em T2 em hipocampo direito, de natureza a esclarecer. Os demais seis pacientes não exibiam alterações no exame de RM de encéfalo.

Todos os pacientes inclusos foram submetidos a pelo menos um exame eletroneuromiográfico. Vinte e seis (65\%) exames apresentavam alterações sugestivas de um processo desmielinizantes, oito $(20 \%)$ revelaram achados de uma neuropatia primariamente axonal, enquanto cinco (12,5\%) exibiam achados eletromiográficos indeterminados, não sendo possível classificar entre um processo primariamente desmielinizante ou axonal. Um paciente apresentou redução da amplitude dos potenciais motores dos nervos faciais à condução motora e estudo do reflexo do piscamento alterado, com respostas R1 e R2 ausentes, tendo o diagnóstico de PNC. As eletroneuromiografias foram realizadas com $8,8 \pm 6,7$ dias do início do quadro motor. 


\subsection{DIAGNÓSTICO, TRATAMENTO E DESFECHO CLÍNICO}

O diagnóstico de SGB foi definido para cada paciente de acordo com os achados clínicos, laboratoriais e eletroneuromiográficos. Vinte (50\%) pacientes apresentavam o diagnóstico compatível com PIDA, sete (17,5\%) o diagnóstico de AMAN e três $(7,5 \%)$ o diagnóstico de SGB paraparético. Um paciente recebeu o diagnóstico de polineurite craniana (PNC). Cinco casos (12,5\%) tinham um padrão clínico de sobreposição incompleta. Desses, uma criança apresentou paraparesia arreflexa e ptose palpebral bilateral, padrão eletrofisiológico de um processo axonal e quadro compatível de sobreposição incompleta SMF e SGB-P. As outras quatro crianças apresentaram um quadro sugestivo de encefaloneurite: três apresentaram um quadro de fraqueza arreflexa (tetraparesia em duas e paraparesia em uma) associado à alteração do nível de consciência e padrão eletrofisiológico desmielinizante, e uma apresentou uma tetraparesia proximal e simétrica, ataxia mista e nistagmos, também com padrão eletrofisiológico desmielinizante. Quatro casos (10\%) exibiam um padrão clínico e eletroneuromiográfico que não nos permitiu classificá-los entre AIDP e AMAN. Somente em um desses casos a ENMG foi repetida após quatorze dias do início do quadro, e ainda assim manteve alterações inespecíficas para um processo mielínico ou axonal. Os dados relativos ao diagnóstico e variantes estão sumarizados no Gráfico 2. 


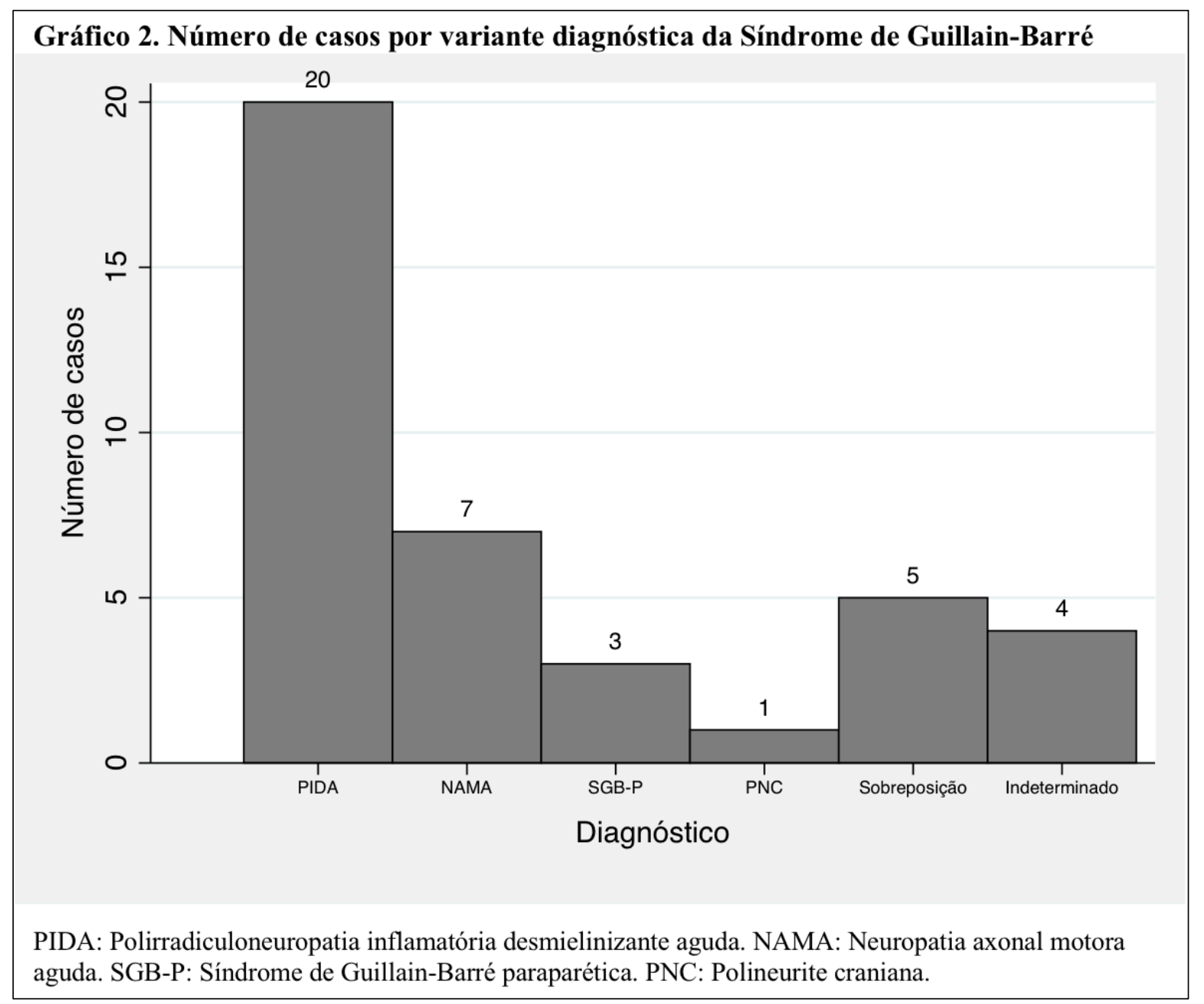

Exceto dois, os pacientes receberam tratamento medicamentoso além das medidas de suporte. Em sua maioria (34 casos, $85 \%$ ), a terapia utilizada foi a IgIV. Três pacientes $(7,5 \%)$ receberam tratamento combinado de IgIV e corticoides, e um paciente recebeu somente corticoides. Este último recebeu a corticoterapia em outro serviço e por ter sido admitido no HCFMRP-USP em melhora clínica, foi optado por não tratar com IgIV. Plasmaférese não foi utilizada como modalidade de tratamento em nenhum dos casos. A terapêutica foi instituída com 7,5 \pm 5,4 dias do início dos sintomas. Em sete casos $(17,5 \%)$ inicialmente tratados com IgIV, foi optado pelo re-tratamento com uma segunda dose de ataque de IgIV, após ter sido observada pouca resposta à dose inicial e manutenção do comprometimento funcional. Em todos os casos re-tratados, observou-se uma melhora gradual da força após o re-tratamento, sendo que todos apresentaram doença de curso monofásico. 
Os desfechos funcionais aferidos através da escala funcional de Hughes (HUGHES et al., 1992) estavam disponíveis para 38 dos 40 pacientes na admissão, 36 dos 40 no nadir e 29 dos 40 na alta hospitalar. Tanto na admissão hospitalar quanto no nadir neurológico, a maioria dos pacientes estava incapaz de deambular (21 pacientes com classe funcional 4 ou 5 na escala funcional de Hughes). Na alta hospitalar, somente dez pacientes mantinham esse grau de disfunção. Cinco pacientes necessitaram de intubação orotraqueal ao longo da evolução clínica (classe funcional 5 da escala funcional de Hughes), sendo que três foram admitidos intubados e dois tiveram alta hospitalar para um hospital secundário ainda em ventilação mecânica. O Gráfico 3 sumariza a evolução da disfunção dos 28 pacientes em que se tinha o registro da escala funcional na admissão, no nadir e na alta hospitalar. O tempo médio de intubação orotraqueal foi de 19,3 $\pm 8,3$ dias. O número total de dias de internação variou de três a setenta e um dias, com uma média de 13,7 \pm 12,8 dias (mediana 9 dias, IIQ 7 - 16 dias).

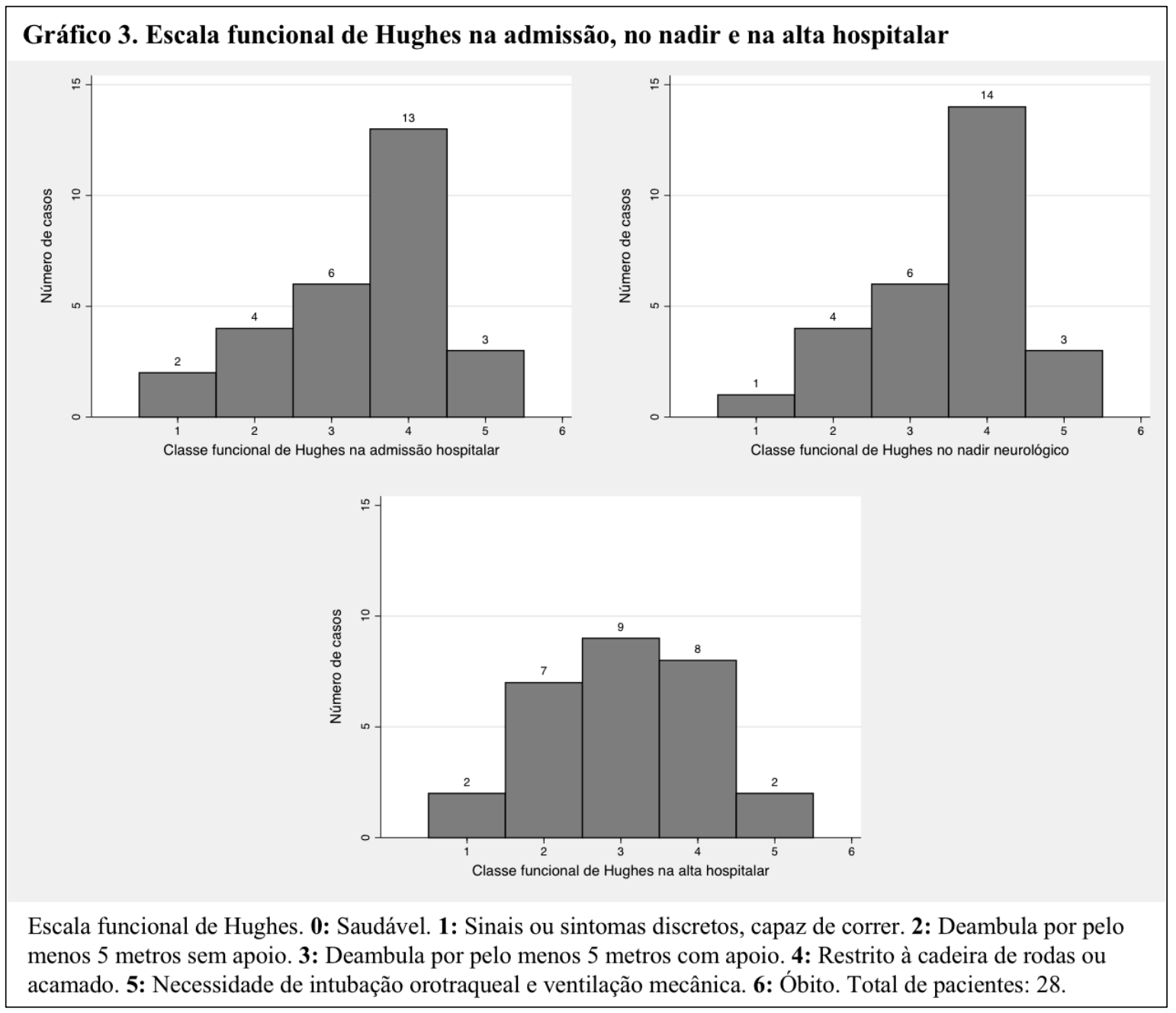




\section{DISCUSSÃO}

Este estudo descreve a experiência de dezesseis anos do serviço de neurologia do HCFMRP-USP e incluiu quarenta crianças de oito meses a quinze anos de idade. Meninos representaram 1,35 vezes mais casos do que meninas, compatível com a maior incidência da SGB no sexo masculino (SEJVAR et al., 2011). O número de casos que apresentaram assimetria durante a evolução clínica foi considerável, representando 15\% dos casos. Entretanto, somente dois pacientes apresentavam algum tipo de assimetria no exame neurológico à admissão hospitalar e um paciente teve uma progressão assimétrica da força muscular após a internação. As outras três crianças exibiram quadros que inicialmente eram assimétricos, mas que progrediram para simetria antes da primeira avaliação. Além dos casos assimétricos, também foi observado um número elevado de casos com acometimento segmentar ou focal na primeira avaliação. De um total de oito, cinco pacientes tinham uma paraparesia simétrica, um tinha fraqueza proximal, um tinha fraqueza distal, e um tinha um quadro motor focal acometendo somente nervos cranianos.

Como somente dois pacientes tinham clínica assimétrica na admissão hospitalar, optou-se por agrupar esses pacientes com os oito que apresentaram acometimento motor segmentar e focal, como um grupo composto por apresentações consideradas atípicas na SGB. A presença de quadro motor assimétrico e segmentar não estava associada ao sexo, presença de eventos precedentes, padrão eletroneuromiográfico, características clinico-laboratoriais ou desfechos funcionais. Os casos assimétricos e segmentares apresentaram uma correlação significativa $(\mathrm{p}=0,004)$ com a progressão estática dos sintomas motores, demonstrando que os pacientes que exibem acometimento motor assimétrico ou segmentar têm uma tendência maior a permanecer dessa maneira durante a evolução clínica. Observou-se também uma tendência $(\mathrm{p}=0,08)$ dos pacientes com exame assimétrico serem mais jovens, mas essa diferença não foi significativa.

Esses resultados são condizentes com outros estudos que haviam relatado uma maior proporção de achados motores atípicos na SGB em crianças. Karimzadeh e colaboradores descreveram um total de $24,2 \%$ de pacientes com sintomas atípicos de $\mathrm{SGB}$, incluindo fraqueza exclusiva de membros superiores em $3 \%$ e fraqueza proximal 
em 9\% (KARIMZADEH et al., 2012). Em uma série de 61 crianças com idades entre sete meses a treze anos, Linden et al. notaram uma prevalência de $18 \%$ de casos com fraqueza crural exclusiva e 4,9\% com uma distribuição assimétrica dos déficits no início do quadro e que regrediu ou se atenuou ao longo da evolução clínica (LINDEN et al., 2010). Pérez-Lledó e colaboradores também observaram um acometimento exclusivo de membros inferiores em $25 \%$, numa série de oito pacientes, com um paciente iniciando o quadro com hemiparesia esquerda que progrediu para tetraparesia simétrica no nadir (PÉREZ-LLEDÓ et al., 2012). Por outro lado, a prevalência de assimetria motora em adultos parece ser menor do que em crianças. Fokke et al. observaram, em uma coorte de 567 pacientes com mais de dezoito anos e diagnóstico de SGB, que somente 1\% (5 de 486 casos) apresentavam algum tipo de assimetria motora à admissão hospitalar e apenas um paciente teve um quadro de monoparesia isolada (FOKKE et al., 2014).

Outro achado relevante do exame físico na admissão foi uma elevada proporção de pacientes com reflexos miotáticos preservados $(27,5 \%)$ e um paciente com hiperreflexia. A maioria desses pacientes manteve o padrão de preservação de reflexos mesmo durante o nadir. A alta prevalência de pacientes com reflexos preservados em membros paréticos é um dado relevante, uma vez que a redução ou abolição dos reflexos profundos é um sinal cardinal na SGB. Esses achados contrastam com outros estudos que encontraram arreflexia ou hiporreflexia em praticamente todos os pacientes (GUPTA et al., 2008; HICKS et al., 2010; LINDEN et al., 2010; YE et al., 2014; KUMAR et al., 2015). Entretanto, Yuki e colaboradores demonstraram que até 10\% dos pacientes com SGB podem apresentar-se com reflexos normais ou aumentados, e que estes tinham mais frequentemente anticorpos contra os gangliosídeos GM1, GM1b, GD1a, ou GalNAc-GD1a (YUKI et al., 2012). Na maioria dos casos (65\%), a progressão dos sintomas motores foi ascendente, enquanto que $15 \%$ dos pacientes apresentaram uma progressão descendente dos sintomas. Esses achados são importantes, uma vez que a progressão descendente no eixo craniocaudal é considerada atípica e pode sugerir outros diagnósticos diferenciais, como o botulismo (JONES, 2000). Karimzadeh e colaboradores também notaram uma proporção significativa de casos com fraqueza descendente, correspondendo a 15,2\% dos casos (KARIMZADEH et al., 2012). 
Entre os principais sintomas percebidos inicialmente pelo paciente ou cuidador, fraqueza apendicular foi a mais comum (60\%), seguido de dificuldade de marcha em $55 \%$ e dor em $52,5 \%$ dos casos. Além disso, a prevalência de cefaleia como sintoma inicial foi considerável, acometendo 15\% dos pacientes. Dor também foi um achado de exame físico muito prevalente $(42,5 \%)$, embora o exame de sensibilidade estivesse normal em torno de dois terços dos casos. Esses dados estão em concordância com o quadro clínico da SGB em crianças, em que dor é sintoma muito prevalente e muitas vezes é a queixa principal do quadro (BRADSHAW e JONES, 1992; DELANOE et al., 1998; NGUYEN et al., 1999; KORINTHENBERG et al., 2007; DEVOS et al., 2013). $\mathrm{O}$ acometimento de nervos cranianos é mais comum em crianças do que em adultos, podendo estar presente em até metade dos pacientes, e o nervo facial é o mais comumente afetado (KORINTHENBERG et al., 2007; KOUL e ALFUTAISI, 2008; HICKS et al., 2010). Consistentemente, observamos que um total de $47,5 \%$ das crianças apresentava algum tipo de disfunção de nervos cranianos. Entre os nervos cranianos, a paralisia facial bilateral foi o achado mais frequente $(32,5 \%)$, seguido de fraqueza bulbar $(17,5 \%)$ e alteração da motricidade ocular ou ptose palpebral (17,5\%).

A dissociação albuminocitológica no exame de LCR nem sempre está presente, especialmente na primeira semana de evolução. Pleiocitose discreta $\left(\begin{array}{lll}10 & \text { a } & 50\end{array}\right.$ leucócitos $/ \mathrm{mm}^{3}$ ) pode ser eventualmente encontrada, porém concentrações de leucócitos maiores que $50 / \mathrm{mm}^{3}$ são atípicas e devem levantar suspeitas para outros diagnósticos como poliomielite, enteroviroses e infecção pelo HIV (BRADSHAW e JONES, 1992; DELANOE et al., 1998). Nesta série, $47,2 \%$ das crianças que foram submetidas à coleta do LCR apresentavam dissociação albuminocitológica no primeiro exame de punção lombar e nenhuma apresentou celularidade maior que 50 leucócitos $/ \mathrm{mm}^{3}$. O exame de RM foi realizado em casos de dúvida diagnóstica e para exclusão de diagnósticos diferenciais. Dos cinco pacientes submetidos à RM de coluna, dois demonstraram captação de contraste em raízes, um achado comum na SGB em crianças (YIKILMAZ et al., 2010; SMITH et al., 2014). Em contrapartida, todas as nove crianças submetidas à RM de encéfalo exibiam o exame normal ou somente com alterações inespecíficas.

Todos os nossos pacientes foram submetidos a pelo menos um exame eletroneuromiográfico. Em $65 \%$ dos casos, a eletroneuromiografia apresentava 
alterações sugestivas de um processo desmielinizante, $20 \%$ apresentavam achados de uma neuropatia primariamente axonal, e 12,5\% exibiam achados eletromiográficos indeterminados. A criança que recebeu o diagnóstico de polineurite craniana apresentou uma eletroneuromiografia com redução da amplitude dos potenciais motores dos nervos faciais à condução motora e estudo do reflexo do piscamento alterado, com respostas R1 e R2 ausentes. Os pacientes receberam o diagnóstico final de PIDA em 50\% dos casos, AMAN em 17,5\% e SGB paraparético em 7,5\%. Nós achamos uma proporção de $12,5 \%$ de casos compatíveis com a sobreposição clínica: um caso de sobreposição SMF e SGB-P e outros quatro casos com um quadro sugestivo de encefaloneurite. Esses achados são consistentes com a distribuição das variantes clínicas e resultados eletrofisiológicos delineados em outros estudos (BRADSHAW e JONES, 1992; DELANOE et al., 1998; KORINTHENBERG et al., 2007; GUPTA et al., 2008; HICKS et al., 2010) e denotam que casos de sobreposição clínica não são incomuns em crianças. Em 12,5\% dos casos, não foi possível classificar o padrão eletrofisiológico em primariamente desmielinizante ou axonal. Desse modo, esses casos acabaram sendo classificados como indeterminados. Infelizmente, somente um desses pacientes (Anexo 4, caso I) repetiu a ENMG em um segundo tempo, e ainda assim as alterações encontradas foram inespecíficas.

A maioria das crianças deste estudo foram tratadas com IgIV. Em 17,5\% dos casos, optou-se pelo re-tratamento com uma segunda dose de ataque de IgIV após não ter sido observada uma resposta clínica satisfatória à primeira infusão. Esses pacientes apresentaram uma melhora significativa após a segunda dose. A falha terapêutica à IgIV em alguns casos pode estar relacionada ao metabolismo da medicação e o reforço com uma segunda dose muitas vezes é indicado (HUGHES et al., 2014; GODOY e RABINSTEIN, 2015). A maioria dos pacientes (52,5\%) foi admitida sem conseguir deambular, efetivamente restrita à cadeira de rodas ou acamada (escala funcional de Hughes 4 ou 5). No nadir, 12,5\% necessitavam de suporte ventilatório. De maneira geral, os pacientes apresentaram uma recuperação significativa após o tratamento e na alta, três quartos dos pacientes já conseguiam deambular, com ou sem auxílio. Somente dois pacientes receberam alta ainda em suporte ventilatório e nenhum paciente faleceu, 
resultados que estão em concordância com o melhor prognóstico da SGB em crianças (BRADSHAW e JONES, 1992; DELANOE et al., 1998; DEVOS et al., 2013).

O presente estudo tem algumas limitações. Em se tratando de um estudo retrospectivo, o controle de variáveis de confusão é limitado. A análise estatística foi realizada com controle para variáveis como sexo e idade, através de regressão logística multivariada, mas devido à amostra relativamente pequena, o controle para mais variáveis de confusão não pôde ser feito. Além disso, embora tenha sido observado uma tendência de os pacientes com assimetrias no exame físico serem mais jovens, uma amostragem maior seria necessária para confirmar essa tendência. A revisão de prontuários, a despeito da utilização de um questionário objetivo, teve que lidar com dados faltantes e utilização de registros antigos não otimizados para pesquisa, o que impediu uma análise mais completa do escore de força muscular da MRC e dos dados relativos a desfechos funcionais na admissão, nadir e alta hospitalar. Ainda assim, a ampla disponibilidade de dados disponíveis nos prontuários, assim como a facilidade de acesso a eles e à objetividade do questionário resultou em um baixo índice de dados faltantes para as demais variáveis. 


\section{CONCLUSÃO}

Em conclusão, os resultados deste estudo demonstram que quadros segmentares e assimétricos não são incomuns na SGB em crianças. As crianças que apresentaram essas assimetrias e quadros focais exibiram uma tendência significativa a permanecer com essas alterações ao longo de todo o curso clínico, em sua maioria. Somente menos da metade eventualmente progrediu para um quadro motor generalizado e simétrico. Além disso, percebeu-se um percentual significativo de pacientes com reflexos preservados, mesmo durante o nadir neurológico e $15 \%$ dos pacientes apresentaram uma progressão descendente dos déficits neurológicos. Tais achados indicam que os critérios diagnósticos da SGB devem ser considerados com cautela na população infantil e que a presença de assimetria persistente, a preservação de reflexos e o caráter descendente dos déficits, dentro do contexto clínico adequado, não excluem necessariamente o diagnóstico de SGB. Por fim, mais estudos são necessários para confirmar esses resultados e coortes prospectivas, com análises sistemáticas, poderiam contribuir para expansão do espectro clínico e diagnóstico da SGB em crianças. 


\section{REFERÊNCIAS}

ASBURY, A. K.; CORNBLATH, D. R. Assessment of current diagnostic criteria for Guillain-Barré syndrome. Ann Neurol, v. 27 Suppl, p. S21-4, 1990.

BRADSHAW, D. Y.; JONES, H. R., JR. Guillain-Barré syndrome in children: clinical course, electrodiagnosis, and prognosis. Muscle Nerve, v. 15, n. 4, p. 500-6, 1992.

CHANSON, J. B.; ECHANIZ-LAGUNA, A. Early electrodiagnostic abnormalities in acute inflammatory demyelinating polyneuropathy: a retrospective study of 58 patients. Clin Neurophysiol, v. 125, n. 9, p. 1900-5, 2014.

DELANOE, C. et al. Acute inflammatory demyelinating polyradiculopathy in children: clinical and electrodiagnostic studies. Ann Neurol, v. 44, n. 3, p. 350-6, 1998.

DEVOS, D. et al. Guillain-Barré syndrome during childhood: particular clinical and electrophysiological features. Muscle Nerve, v. 48, n. 2, p. 247-51, 2013.

DIAS-TOSTA, E.; KÜCKELHAUS, C. S. Guillain-Barré syndrome in a population less than 15 years old in Brazil. Arq Neuropsiquiatr, v. 60 (2-B), p. 367-73, 2002.

FOKKE, C. et al. Diagnosis of Guillain-Barré syndrome and validation of Brighton criteria. Brain, v. 137, n. Pt 1, p. 33-43, 2014.

GODOY, D. A.; RABINSTEIN, A. Is a second cycle of immunoglobulin justified in axonal forms of Guillain-Barré syndrome? Arq Neuropsiquiatr, v. 73, n. 10, p. 848$51,2015$.

GRIFFIN, J. W. et al. Guillain-Barré syndrome in northern China. The spectrum of neuropathological changes in clinically defined cases. Brain, v. 118, p. 577-95, 1995.

GUPTA, D. et al. Electrodiagnostic and clinical aspects of Guillain-Barré syndrome: an analysis of 142 cases. J Clin Neuromuscul Dis, v. 10, n. 2, p. 42-51, 2008.

HAHN, A. F. Guillain-Barré syndrome. Lancet, v. 352, n. 9128, p. 635-41, 1998.

HICKS, C. W. et al. A clinical picture of Guillain-Barré syndrome in children in the United States. J Child Neurol, v. 25, n. 12, p. 1504-10, 2010. 
HUGHES, R. et al. Subacute idiopathic demyelinating polyradiculoneuropathy. Arch Neurol, v. 49, n. 6, p. 612-6, 1992.

HUGHES, R. A.; SWAN, A. V.; VAN DOORN, P. A. Intravenous immunoglobulin for Guillain-Barré syndrome. Cochrane Database Syst Rev, n. 9, p. Cd002063, 2014.

JONES, H. R. Guillain-Barré syndrome: perspectives with infants and children. Semin Pediatr Neurol, v. 7, n. 2, p. 91-102, 2000.

KALITA, J. et al. Guillain-Barré syndrome: subtypes and predictors of outcome from India. J Peripher Nerv Syst, v. 19, n. 1, p. 36-43, 2014.

KARIMZADEH, P. et al. Atypical findings of Guillain-Barré syndrome in children. Iran J Child Neurol, v. 6, n. 4, p. 17-22, 2012.

KLEYWEG, R. P.; VANDERMECHE, F. G. A.; SCHMITZ, P. I. M. Interobserver agreement in the assessment of muscle strength and functional abilities in Guillain-Barré syndrome. Muscle \& Nerve, v. 14, n. 11, p. 1103-1109, 1991.

KORINTHENBERG, R. Acute polyradiculoneuritis: Guillain-Barré syndrome. Handb Clin Neurol, v. 112, p. 1157-62, 2013.

KORINTHENBERG, R.; SCHESSL, J.; KIRSCHNER, J. Clinical presentation and course of childhood Guillain-Barré syndrome: a prospective multicentre study. Neuropediatrics, v. 38, n. 1, p. 10-7, 2007.

KOUL, R. L.; ALFUTAISI, A. Prospective study of children with Guillain-Barré syndrome. Indian J Pediatr, v. 75, n. 8, p. 787-90, 2008.

KUMAR, M. et al. Guillain-Barré syndrome: a clinical study of twenty children. J Clin Diagn Res, v. 9, n. 1, p. Sc09-12, 2015.

LEE, J. H.; SUNG, I. Y.; REW, I. S. Clinical presentation and prognosis of childhood Guillain-Barré syndrome. J Paediatr Child Health, v. 44, n. 7-8, p. 449-54, 2008.

LIN, J. J. et al. Clinical variants of Guillain-Barré syndrome in children. Pediatr Neurol, v. 47, n. 2, p. 91-6, 2012. 
LINDEN, V. et al. Guillain-Barré syndrome in children: clinic, laboratorial and epidemiologic study of 61 patients. Arq Neuropsiquiatr, v. 68, n. 1, p. 12-7, 2010.

MULKEY, S. B. et al. Nerve root enhancement on spinal MRI in pediatric GuillainBarré syndrome. Pediatr Neurol, v. 43, n. 4, p. 263-9, 2010.

NGUYEN, D. K.; AGENARIOTI-BÉLANGER, S.; VANASSE, M. Pain and the Guillain-Barré syndrome in children under 6 years old. J Pediatr, v. 134, n. 6, p. 7736, 1999.

PÉREZ-LLEDÓ, E.; DÍAZ-VICO, A.; GÓMEZ-GOSÁlVEZ, F. A. Síndrome de Guillain-Barré: presentación clínica y evolución en menores de 6 años de edad. Anales de Pediatría, v. 76, n. 2, p. 69-76, 2012.

RAJABALLY, Y. A. Treatment of Guillain-Barré syndrome: a review. Inflamm Allergy Drug Targets, v. 11, n. 4, p. 330-4, 2012.

RAPHAEL, J. C. et al. Plasma exchange for Guillain-Barré syndrome. Cochrane Database Syst Rev, n. 7, p. Cd001798, 2012.

ROODBOL, J. et al. Recognizing Guillain-Barré syndrome in preschool children. Neurology, v. 76, n. 9, p. 807-10, 2011.

SEJVAR, J. J. et al. Population incidence of Guillain-Barré syndrome: a systematic review and meta-analysis. Neuroepidemiology, v. 36, n. 2, p. 123-33, 2011.

SHAHRIZAILA, N.; YUKI, N. Bickerstaff brainstem encephalitis and Fisher syndrome: anti-GQ1b antibody syndrome. J Neurol Neurosurg Psychiatry, v. 84, n. 5, p. 576-83, 2013.

SMITH, N.; PEREIRA, J.; GRATTAN-SMITH, P. Investigation of suspected GuillainBarré syndrome in childhood: what is the role for gadolinium enhanced magnetic resonance imaging of the spine? J Paediatr Child Health, v. 50, n. 10, p. E72-6, 2014.

TANG, J. et al. Guillain-Barré syndrome in Chinese children: a retrospective analysis. Pediatr Neurol, v. 45, n. 4, p. 233-7, 2011.

UNCINI, A.; KUWABARA, S. Electrodiagnostic criteria for Guillain-Barré syndrome: a critical revision and the need for an update. Clin Neurophysiol, v. 123, n. 8, p. 1487$95,2012$. 
VAN DOORN, P. A. Diagnosis, treatment and prognosis of Guillain-Barré syndrome (GBS). Presse Med, v. 42, n. 6 Pt 2, p. e193-201, 2013.

WAKERLEY, B. R. et al. Guillain-Barré and Miller Fisher syndromes--new diagnostic classification. Nat Rev Neurol, v. 10, n. 9, p. 537-44, 2014.

WILLISON, H. J.; JACOBS, B. C.; VAN DOORN, P. A. Guillain-Barré syndrome. Lancet, v. 388, n. 10045, p. 717-27, 2016.

YE, Y. Q. et al. Clinical and electrophysiologic features of childhood Guillain-Barré syndrome in Northeast China. J Formos Med Assoc, v. 113, n. 9, p. 634-9, 2014.

YIKILMAZ, A. et al. Magnetic resonance imaging of childhood Guillain-Barré syndrome. Childs Nerv Syst, v. 26, n. 8, p. 1103-8, 2010.

YUKI, N.; HARTUNG, H. P. Guillain-Barré syndrome. N Engl J Med, v. 366, n. 24, p. 2294-304, 2012.

YUKI, N. et al. Guillain-Barré syndrome associated with normal or exaggerated tendon reflexes. J Neurol, v. 259, n. 6, p. 1181-90, 2012.

YUKI, N. Carbohydrate mimicry between human ganglioside GM1 and Campylobacter jejuni lipooligosaccharide causes Guillain-Barré syndrome. Proc Natl Acad Sci U S A, v. 101, n. 31, p. 11404-9, 2004.

ZUCCOLI, G. et al. Redefining the Guillain-Barré spectrum in children: neuroimaging findings of cranial nerve involvement. AJNR Am J Neuroradiol, v. 32, n. 4, p. 63942, 2011. 


\section{ANEXOS}

\section{ANEXO 1 - FORMULÁRIO DE PESQUISA}

Protocolo de pesquisa desenvolvido como parte do Projeto de Mestrado Profissional - HCFMRP-USP

Responsáveis: Pedro Henrique Marte de Arruda Sampaio (pesquisador), Amilton Antunes Barreira (orientador).

.Registro HC-FMRP:

.Nome (iniciais):

.Sexo: Masculino Feminino

.Data do nascimento:

.Idade no início do quadro (anos completos):

.Data da admissão HC-FMRP-USP:

.Evento precedente (marcar os eventos precedentes que se apliquem)

Infecção de vias aéreas (superiores ou inferiores)

Diarreia

Evento mononucleose-like

__ Evento dengue-like

__ Evento febril não-especificado - inclui eventos febris que não se encaixem nas categorias acima

__ Vacinação (especificar no item seguinte)

__ Cirurgia (especificar no item seguinte)

__ Outro (especificar):

Evento precedente - tempo em dias do início dos sintomas: 


\section{.Primeiro(s) sintomas(s):}

Fraqueza apendicular

Dificuldade de marcha

_ Desequilíbrio ou alteração de coordenação

_ Dor ou parestesias dolorosas

__ Cefaleia

__ Parestesias não dolorosas, dormência ou anestesia

Envolvimento motor de nervos cranianos

_. Alterações esfincterianas

__ Alteração do nível de consciência

__ Outro (especificar):

.Número de dias entre o início dos sintomas motores e a primeira avaliação no HCFMRP-USP:

.Admissão: Escore somado da MRC - lado direito (0-30):

.Admissão: Escore somado da MRC - lado esquerdo (0-30):

.Admissão: Escore somado do MRC total (0- 60):

.Motricidade apendicular na admissão:

_ Sem fraqueza apendicular

_ Tetraparesia simétrica

_ Tetraparesia assimétrica

_ Tetraparesia de comprometimento proximal (poupa distal)

_ Tetraparesia de comprometimento distal (poupa proximal)

_ Paraparesia (poupa membros superiores)

__ Paresia de membros superiores (poupa membros inferiores)

_ Hemiparesia (poupa o dimidio contralateral)

_ Hemiparesia alterna (membro superior + membro inferior contralateral)

_ Monoparesia de membro inferior (poupa os demais membros)

_ Monoparesia de membro superior (poupa os demais membros) 


\section{Reflexos na admissão:}

Membros afetados arreflexos ou hiporreflexos - Arreflexia (grau 0) ou hiporreflexia (grau 1)

_ Membros afetados normorreflexos - Normorreflexia (grau 2 ou grau 3 sem piramidalismo)

_ Membros afetados hiperreflexos - Hiperreflexia (grau 4 ou grau $3 \mathrm{com}$ piramidalismo)

Membros poupados arreflexos ou hiporreflexos - Arreflexia (grau 0) ou hiporreflexia (grau 1)

_ Membros poupados normorreflexos - Normorreflexia (grau 2 ou grau 3 sem piramidalismo)

_ Membros poupados hiperreflexos - Hiperreflexia (grau 4 ou grau 3 com piramidalismo)

\section{.Dor e parestesias dolorosas:}

Ausência de dor

_ Presença de dor

\section{Alterações sensitivas:}

_ Exame de sensibilidade T-D, vibratório e posição segmentar normal Exame de sensibilidade T-D alterado

__ Exame de sensibilidade vibratória ou posição segmentar alterada

__ Exame de sensibilidade T-D e vibratório ou posição segmentar alterados

_ Indeterminado - paciente não colaborativo

\section{Ataxia:}

Sem ataxia

Ataxia cerebelar

Ataxia sensitiva

Ataxia mista (cerebelar + sensitiva)

\section{.Nervo facial (Nervo craniano VII):}

__ Sem acometimento do nervo facial (VII)

__ Paresia facial periférica

_ Paresia facial central 
.Oftalmoparesia (Nervos cranianos III, IV, VI):

_ Ausente

Presente

.Envolvimento bulbar (nervos cranianos IX, X, XI, XII):

Ausência de envolvimento bulbar

__ Presença de envolvimento bulbar

.Alteração esfincteriana:

Ausente

_ Presente

.Disautonomia:

Ausente
P Presente

.Alteração da consciência:

_ Ausente

Presente

.Sinais meníngeos:

Ausente

Presente

.Exame motor assimétrico ou focal na admissão:

_ Simétrico

_ Assimétrico

Focal

.Escala funcional de Hughes na admissão:

$0-1-2-3-{ }^{4}-{ }^{2}-6_{-}$

.Nadir motor MRC-SS - lado direito (0-30):

.Nadir motor MRC-SS - lado esquerdo (0-30): 
.Nadir motor MRC-SS - total (0 - 60):

\section{.Nadir motor - motricidade apendicular:}

_ Sem fraqueza apendicular

Tetraparesia simétrica

Tetraparesia assimétrica

Tetraparesia de comprometimento proximal (poupa distal)

Tetraparesia de comprometimento distal (poupa proximal)

Paraparesia (poupa membros superiores)

_ Paresia de membros superiores (poupa membros inferiores)

__ Hemiparesia (poupa o dimidio contralateral)

_ Hemiparesia alterna (membro superior + membro inferior contralateral)

_ Monoparesia de membro inferior (poupa os demais membros)

__ Monoparesia de membro superior (poupa os demais membros)

.Escala funcional de Hughes no nadir motor:

$0-1-2-3-4-5-6$

\section{Nadir motor - Reflexos:}

_ Membros afetados arreflexos ou hiporreflexos - Arreflexia (grau 0) ou hiporreflexia (grau 1)

__ Membros afetados normorreflexos - Normorreflexia (grau 2 ou grau 3 sem piramidalismo)

_ Membros afetados hiperreflexos - Hiperreflexia (grau 4 ou grau 3 com piramidalismo)

_ Membros poupados arreflexos ou hiporreflexos - Arreflexia (grau 0) ou hiporreflexia (grau 1)

_ Membros poupados normorreflexos - Normorreflexia (grau 2 ou grau 3 sem piramidalismo)

_ Membros poupados hiperreflexos - Hiperreflexia (grau 4 ou grau $3 \mathrm{com}$ piramidalismo)

.Nadir - Disautonomia:

Ausente

Presente 
.Número de dias entre o início dos sintomas motores e o nadir neurológico:

.Número de dias entre a primeira avaliação e o nadir neurológico:

.Número de dias do platô neurológico:

.Nadir - progressão para sintomas simétricos ou generalizados?

__ Manteve assimetria

Manteve focal

Tornou-se simétrico

Tornou-se generalizado

Já era simétrico/generalizado

.Nadir - tempo em dias para se tornar simétrico ou generalizado:

.Progressão dos déficits neurológicos no eixo crâniocaudal:

__ Estático

Ascendente

Descendente

.LCR celuraridade (Número de leucócitos por mm3 no primeiro LCR realizado):

.LCR proteínas (Proteínas liquóricas em mg/dl no primeiro LCR realizado):

.LCR - dissociação albuminocitológica:

_ Não preenche critérios

Preenche critérios

.LCR 2- tempo em dias entre o início dos sintomas motores e a coleta do segundo LCR:

.LCR 2 celuraridade (Número de leucócitos por mm3 no segundo LCR realizado):

.LCR 2 proteínas (Proteínas liquóricas em mg/dl no segundo LCR realizado): 
.LCR 2- dissociação albuminocitológica:

_ Não preenche critérios

_ Preenche critérios

.LCR 2- tempo em dias entre o início dos sintomas motores e a coleta do segundo LCR:

RM coluna:

Exame normal

__ Lesão medular desmielinizante

_ Captação de contraste radicular ou plexo

__ Espessamento de raízes ou plexo

_ Outros:

\section{.RM encéfalo:}

__ Exame normal

__ Lesão desmielinizante infratentorial

__ Lesão desmielinizante supratentorial

__ Lesões expansivas

__ Lesão isquêmica

_ Outros:

.Primeira ENMG - laudo:

Desmielinizante

Axonal

__ Alterações mistas

__ Indeterminado

__ Inexcitável

_ Outros:

.Primeira ENMG - tempo em dias entre a realização e o início do quadro motor: 
.Segunda ENMG - laudo:

Desmielinizante

Axonal

Alterações mistas

Indeterminado

Inexcitável

Outros:

.Segunda ENMG - tempo em dias entre a realização e o início do quadro motor:

.Diagnóstico - subtipo de SGB:

_ AIDP clássico

AMAN

_ AMSAN

_ SGB paraparético

__ Paresia bifacial com parestesias

__ Fraqueza faringo-cervico-braquial

__ Polineurite craniana

__ Forma disautonômica pura

__ Síndrome de Fisher (forma completa ou incompleta)

__ Síndrome de Bickerstaff (forma completa ou incompleta)

_ Outro:

\section{.Tratamento:}

_ Medidas de suporte

__ Imunoglobulina humana

__ Plasmaférese

_ Corticoides

_ Combinado (especificar):

__ Outro:

.Tratamento - número de dias entre o início dos sintomas motores e o início do tratamento: 
.Flutuações clínicas após o tratamento:

Presente

_ Ausente

.Re-tratamento:

_ Sem re-tratamento

__ Imunoglobulina humana

__ Plasmaférese

Corticoides

_ Combinado (especificar):

_ Outro:

.Necessidade de ventilação mecânica e IOT/TQT? Não $\operatorname{Sim}$

.Número de dias em IOT/TQT:

.Número de dias de internação hospitalar (total):

.Escala funcional de Hughes na alta hospitalar:
0
1
2
3
4
5
6 
O escore somado da $M R C$ é a soma das escalas $M R C$ de seis grupos musculares:

- Abdutores do ombro

- Flexores do cotovelo

- Extensores do punho

- Flexores do quadril

- Extensores do joelho

- Dorsiflexores dos pés

- Total: $(\quad)$

\begin{tabular}{|c|c|}
\hline Direito ( & ) Esquerdo ( \\
\hline Direito ( & ) Esquerdo ( \\
\hline Direito ( & ) Esquerdo ( \\
\hline Direito ( & ) Esquerdo ( \\
\hline Direito ( & ) Esquerdo ( \\
\hline Direito ( & ) Esquerdo ( \\
\hline
\end{tabular}

O exame é realizado bilateralmente, e cada grupo muscular é graduado de 0 a 5 conforme a graduação abaixo:

0 - Ausência de contração visível

1 - Contração visível sem movimento do membro

2 - Movimento ativo do membro, mas não contra a gravidade

3 - Movimento ativo contra a gravidade completando quase todo o movimento articular 4 - Movimento ativo contra a gravidade e resistência

5 - Força normal

Soma-se o escore de cada grupo muscular. O escore varia de 60 (força muscular normal) até 0 (tetraplegia).

Adaptado de: KLEYWEG, R. P.; VANDERMECHE, F. G. A.; SCHMITZ, P. I. M. Interobserver agreement in the assessment of muscle strength and functional abilities in Guillain-Barré syndrome. Muscle \& Nerve, v. 14, n. 11, p. 1103-1109, Nov 1991. 


\section{ANEXO 3 -ESCALA FUNCIONAL DE HUGHES}

0: Saudável.

1: Sinais ou sintomas discretos, capaz de correr.

2: Deambula por pelo menos 5 metros sem apoio.

3: Deambula por pelo menos 5 metros com apoio.

4: Restrito à cadeira de rodas ou acamado.

5: Necessidade de intubação orotraqueal e ventilação mecânica.

6: Óbito.

Adaptado de: HUGHES, R. et al. Subacute idiopathic demyelinating polyradiculoneuropathy. Arch Neurol, v. 49, n. 6, p. 612-6, Jun 1992. 


\section{ANEXO 4 - CASOS ILUSTRATIVOS DE ASSIMETRIA}

CASO I - Paciente do sexo masculino, de oito meses de idade. Tinha antecedente de exantema febril dezessete dias antes do início dos sintomas e vacinação para pneumococo (vacina pneumocócica 10-valente) onze dias antes. A cuidadora notou incialmente uma fraqueza nos membros e dificuldade para engatinhar e sustentar o tronco. O paciente foi admitido no HCFMRP-USP três dias após o início dos sintomas, apresentando uma tetraparesia assimétrica e arreflexa, de maior comprometimento no membro superior esquerdo. Durante a internação também teve constipação fecal, mas sem outros sinais sugestivos de disautonomia. O primeiro exame de LCR, colhido com quatro dias do início dos sintomas, era normal (1 leucócito por $\mathrm{mm}^{3}$ e proteína de 35 $\mathrm{mg} / \mathrm{dl}$ ). Ao ser repetido no décimo dia de evolução, o LCR exibia 5,66 leucócitos e 49,7 $\mathrm{mg} / \mathrm{dl}$ de proteínas. $\mathrm{O}$ primeiro exame eletroneuromiográfico, realizado com quatro dias do início, evidenciou estudo da condução sensitiva e motora normal, exceto por ausência das ondas $\mathrm{F}$ nos nervos fibulares. A eletroneuromiografia controle, realizada com quatorze dias de evolução, demonstrou a redução das velocidades de condução motora nos nervos tibial e fibular, não sendo detectadas ondas $\mathrm{F}$ no nervo tibial. Devido à assimetria do quadro motor e ao fato de ser uma criança pequena de difícil avaliação neurológica, foi optado por realizar RM de neuroeixo. A RM de encéfalo foi compatível com a normalidade, enquanto que a RM de coluna demonstrou captação de contraste magnético em raízes torácicas e lombossacras. A terapêutica com IgIV foi instituída no dia seguinte à internação hospitalar, após o primeiro exame de LCR e ENMG. O paciente recebeu alta após dezoito dias, em melhora clínica franca e já sem sequelas neurológicas no retorno ambulatorial.

CASO II - Menino de um ano e meio, com antecedente de infecção de vias aéreas há vinte e três dias e vacinação para poliomielite há dezesseis dias do início do quadro. A mãe havia percebido fraqueza em pernas e dificuldade para andar sete dias antes da admissão hospitalar. A criança foi admitida com quadro de arreflexia e paraparesia assimétrica, pior no membro inferior esquerdo e deambulando somente com apoio. A ENMG, realizada com oito dias do início dos sintomas demonstrou um processo desmielinizante. Não foi colhido LCR. O paciente permaneceu com fraqueza assimétrica, exclusivamente nos membros inferiores, ao longo do nadir neurológico. Teve quadro compatível com SGB-P, recebendo IgIV no oitavo dia de evolução. Recebeu alta após cinco dias de internação, em melhora clínica, mas ainda com dificuldade de deambular. No retorno após um ano, vinha sem sequelas motoras perceptíveis no exame.

CASO III - Criança de sete anos, do sexo masculino, sem história de quadro infeccioso ou outro antecedente antes do início do quadro. Paciente percebeu, inicialmente, dor em membros, tendo sido admitido no HCFMRP-USP com tetraparesia simétrica e arreflexa após dois dias. Na primeira avaliação neurológica, o paciente tinha um escore somado da MRC de 47 (24 pontos à direita e 23 pontos à esquerda). Após três dias da admissão, o paciente notou piora da fraqueza, que passou a comprometer mais o lado esquerdo e o impossibilitando de deambular, com um escore somado da MRC neste período de 36 (21 pontos à direita e 15 pontos à esquerda). O LCR foi colhido na data da admissão e 
foi normal (1,6 leucócitos por $\mathrm{mm}^{3}$ e 10,5 $\mathrm{mg} / \mathrm{dl}$ de proteínas). A ENMG demonstrou bloqueios parciais da condução motora (nos nervos mediano e ulnar direitos e nos fibulares e tibiais), mas com velocidade de condução motora e sensitiva normais e preservação das latências distais. Foi programado uma ENMG controle após o tratamento para determinar se esses achados eram secundários a um processo primariamente mielínico ou se era um bloqueio reversível, compatível com os quadros axonais. Entretanto, o paciente não compareceu ao exame e o caso foi classificado com indeterminado neste estudo. A criança foi tratada com IgIV no quarto dia de evolução, apresentando melhora progressiva da força e recebendo alta já capaz de deambular, ainda que com dificuldade.

CASO IV - Jovem de doze anos do sexo feminino, sem antecedentes infecciosos precedendo o início dos sintomas. A paciente percebeu inicialmente cefaleia e fraqueza dos membros superiores assimétrica, sendo admitida no HCFMRP-USP após quatorze dias do início dos sintomas. Durante esse período, a fraqueza progrediu de maneira descendente no eixo craniocaudal, evoluindo para uma tetraparesia simétrica e arreflexa na admissão. A paciente também passou a apresentar diparesia facial de leve intensidade. O LCR, colhido com dezesseis dias de evolução, exibia dissociação albuminocitológica $\left(1,6\right.$ leucócitos por $\mathrm{mm}^{3}$ e proteínas de $\left.114,4 \mathrm{mg} / \mathrm{dl}\right)$. Devido à natureza assimétrica dos sintomas, optou-se pela realização de RM de neuroeixo. A RM de coluna foi compatível com a normalidade e a RM de encéfalo evidenciou somente um hipersinal em T2 na região hipocampal direita, de natureza a esclarecer, mas que na época não foi considerado associado ao quadro de SGB. Foi submetida à ENMG com dezesseis dias do início do quadro, evidenciando um processo desmielinizante. Recebeu tratamento com IgIV após a ENMG e LCR, com melhora progressiva dos sintomas, recebendo alta após oito dias, capaz de andar com dificuldade. No retorno de um ano já não exibia sequelas motoras.

CASO V - Paciente do sexo masculino, quatorze anos. Jovem apresentou, uma semana antes do início dos sintomas, quadro de infecção de vias aéreas e diarreia. Evoluiu com fraqueza do membro inferior esquerdo que ao longo de seis dias progrediu de maneira ascendente para uma tetraparesia simétrica, mas com preservação dos reflexos miotáticos. Foi admitido com um escore somado da MRC de 20 (10 pontos à direita $\mathrm{e}$ 10 pontos à esquerda) e também com hipoestesia dolorosa em membros. O paciente manteve os reflexos preservados durante a evolução clínica. O exame de LCR foi colhido na admissão, seis dias após o início do quadro motor, e revelou-se dissociado (1 leucócito por $\mathrm{mm}^{3}$ e $96 \mathrm{mg} / \mathrm{dl}$ de proteínas). A ENMG foi realizada no dia seguinte, e evidenciou um processo desmielinizante. Foi tratado com IgIV após a ENMG, recebendo alta após oito dias de internação hospitalar. Após um ano do quadro, apresentava déficits motores discretos, mas sem prejuízo para as atividades diárias.

CASO VI - Menina de quatorze anos, abriu o quadro com fraqueza e dor em membro inferior esquerdo, vinte e quatro dias após um episódio de amigdalite bacteriana. A fraqueza progrediu de maneira ascendente, tendo sido admitida após seis dias com tetraparesia simétrica e escore somado da MRC de 45 (23 à direita e 22 à esquerda). A paciente ainda apresentava dor em membros inferiores e tinha os reflexos miotáticos 
preservados. O exame de LCR, realizado em outro serviço com três dias do início do quadro, foi normal (1 leucócito por $\mathrm{mm}^{3}$ e $35 \mathrm{mg} / \mathrm{dl}$ de proteínas). Porém, observou-se a conversão do padrão liquórico na segunda punção, realizada cinco dias depois $(4,6$ leucócitos por $\mathrm{mm}^{3}$ e $58 \mathrm{mg} / \mathrm{dl}$ de proteínas). A paciente foi submetida à ENMG após nove dias do início dos sintomas motores, evidenciando amplitudes reduzidas dos potenciais motores nos nervos fibular direito e tibiais, que não eram suficientes para caracterizar uma neuropatia como desmielinizante ou axonal. A paciente foi classificada como subtipo indeterminado neste estudo por essa razão. Como a paciente já vinha em melhora clínica desde antes da admissão hospitalar, foi optado por não instituir nenhum tratamento além de medidas de suporte clínico. Recebeu alta após três dias de internação hospitalar e, no retorno após quatro meses, já não apresentava mais déficits neurológicos. 


\section{ANEXO 5 - APROVAÇÃO DO COMITÊ DE ÉTICA}

HOSPITAL DAS CLINICAS DA FACULDADE DE MEDICINA DE RIBEIRÃO PRETO DA UNIVERSIDADE DE SÃO PAULO

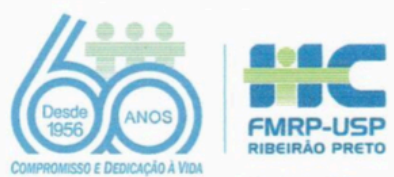

kibeirão Preto. 10 de fevereiro de 2017

Oficio $n^{\circ} 416 / 2017$

$\mathrm{CEP} / \mathrm{MGV}$

Prezados Pesquisadores,

$\bigcirc$ trabalho intitulado "ASSIMETRIAS NO EXAME NEUROLÓGICO DE CRIANÇAS COM SÍNDROME DE GUILLAIN-BARRE”", foi analisado pelo Comitê de Ética em Pesquisa, em sua $443^{a}$ Reunião Ordinária realizada em 06/02/2017 e enquadrado na categoria: APROVADO, bem como a solicitação de dispensa do Termo de Consentimento Livre e Esclarecido, de acordo com o Processo HCRP $\mathrm{n}^{\circ}$ $1028 / 2017$.

Este Comitê segue integralmente a Conferência Internacional de Harmonização de Boas Práticas Clínicas (IGH-GCP), bem como a Resolução $n^{\circ}$ 466/12 CNS/MS.

Lembramos aue devem ser apresentados a este CEP, o Relatório Parcial e o Relatório Final áa pesauisa

Atenciosamente

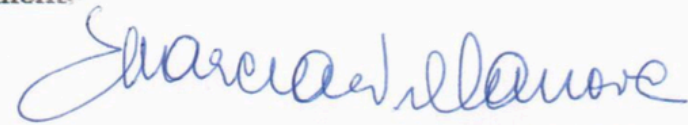

DR ${ }^{a}$. MARCIA GUIMARAES VILLANOVA

Coordenadora do Comitê de Ética em

Pesquisa do HCRP e da FMRP-USP

Ilustríssimos Senhores

PEDRO HENRIQUE MARTE DE ARRUDA SAMPAIO

PROF. DR. AMILTON ANTUNES BARREIRA

Departamento de Neurociências e Ciências do Comportamento

HOSPITAL DAS CLINICAS DA FACULDADE DE MEDICINA DE RIBEIRÃO PRETO DA UNIVERSIDADE DE SÃO PAULO
Campus Universitário - Monte Alegre
Comitê de Ettica em Pesquisa do HCRP e FMRP-USP Campus Universitário - Monte Alegre Comitê de Etica em Pesquisa do HCRP e FMRP-USP 14048-900 Ribeirão Preto SP Registro Plataforma Brasil /CONEP $n^{\circ} 5440$ 